\title{
Spinal Phosphinositide 3-Kinase-Akt-Mammalian Target of Rapamycin Signaling Cascades in Inflammation-Induced Hyperalgesia
}

\author{
Qinghao Xu, ${ }^{1}$ Bethany Fitzsimmons, ${ }^{1}$ Joanne Steinauer, ${ }^{1}$ Audrey 0' Neill, ${ }^{2}$ Alexandra C. Newton, ${ }^{2}$ Xiao-Ying Hua, ${ }^{1}$ and \\ Tony L. Yaksh ${ }^{1,2}$ \\ Departments of ${ }^{1}$ Anesthesiology and ${ }^{2}$ Pharmacology, University of California, San Diego, La Jolla, California 92093
}

\begin{abstract}
Phosphinositide 3-kinase (PI3K), Akt, and their downstream kinase, mammalian target of rapamycin (mTOR), are implicated in neural plasticity. The functional linkages of this signaling cascade in spinal dorsal horn and their role in inflammatory hyperalgesia have not been elucidated. In the present work, we identified the following characteristics of this cascade. (1) Local inflammation led to increase in rat dorsal horn phosphorylation (activation) of Akt (pAkt) and mTOR (pmTOR), as assessed by Western blotting and immunocytochemistry. (2) Increased pAkt and pmTOR were prominent in neurons in laminae I, III, and IV, whereas pmTOR and its downstream targets (pS6, p4EBP) were also observed in glial cells. (3) Intrathecal treatment with inhibitors to PI3K or Akt attenuated Formalin-induced second-phase flinching behavior, as well as carrageenan-induced thermal hyperalgesia and tactile allodynia. (4) Intrathecal rapamycin (an mTORC1 inhibitor) displayed anti-hyperalgesic effect in both inflammatory pain models. Importantly, intrathecal wortmannin at anti-hyperalgesic doses reversed the evoked increase not only in Akt but also in mTORC1 signaling (pS6/p4EBP). (5) pAkt and pmTOR are expressed in neurokinin 1 receptor-positive neurons in laminae I-III after peripheral inflammation. Intrathecal injection of Substance P activated this cascade (increased phosphorylation) and resulted in hyperalgesia, both of which effects were blocked by intrathecal wortmannin and rapamycin. Together, these findings reveal that afferent inputs trigged by peripheral inflammation initiate spinal activation of PI3K-Akt-mTOR signaling pathway, a component of which participates in neuronal circuits of facilitated pain processing.
\end{abstract}

\section{Introduction}

After peripheral tissue injury or inflammation, a pain response can be evoked by a normally innocuous stimulus (allodynia) or an enhanced pain response can be generated by a given noxious stimulus (hyperalgesia). An important component of these exaggerated pain states is facilitation of nociceptive processing as indicated by the enhanced response of spinal dorsal horn projection neurons. Enhanced synaptic transmission is considered to be essential for central sensitization after inflammatory stimuli (Latremoliere and Woolf, 2009). Phosphatidylinositol 3-kinase (PI3K), Akt, and the mammalian target of rapamycin (mTOR) are involved in regulating synaptic plasticity in CNS (Hou and Klann, 2004; Jaworski and Sheng, 2006; Hoeffer and Klann, 2010) and may accordingly play a spinal role in the post-tissue injuryfacilitated state.

PI3K generates phosphatidylinositol $(3,4,5)$-trisphosphate [PtdIns $\left.(3,4,5) \mathrm{P} 3\left(\mathrm{PIP}_{3}\right)\right]$, which recruits cellular proteins containing lipid-binding domains to cell membranes. In this manner, $\mathrm{PIP}_{3}$ regulates the phosphorylation of Akt at Thr308 and

Received April 25, 2010; revised Nov. 25, 2010; accepted Dec. 3, 2010

This work was supported by National Institutes of Health Grants NS 16541 and DA 02110 (T.L.Y.).

Correspondence should be addressed to either Dr. Tony L. Yaksh or Dr. Qinghao Xu, Department of Anesthesiology 0818, University of California, San Diego, 9500 Gilman Drive (CTF C-312), La Jolla, CA 92093. E-mail: tyaksh@ucsd.edu or q1xu@ucsd.edu.

DOI:10.1523/JNEUROSCI.2139-10.2011

Copyright $\odot 2011$ the authors $\quad 0270-6474 / 11 / 312113-12 \$ 15.00 / 0$
Ser473, locking Akt in an active conformation (pAkt) (Pearce et al., 2010). Several lines of evidence suggest an involvement of PI3K-linked cascades in the regulation of dorsal horn hyperexcitability. Thus, activity-dependent increases in Akt activation (as measured by phosphorylation) are seen in DRG and dorsal horn neurons (Zhuang et al., 2004; Pezet et al., 2005; Sun et al., 2006, 2007; Pezet et al., 2008; Shi et al., 2009; Choi et al., 2010), and intrathecal PI3K inhibitors attenuate chemical-evoked (Sun et al., 2006; Pezet et al., 2008; Choi et al., 2010) and nerve injuryevoked (Xu et al., 2007) hypersensitivity.

mTOR, specifically mTORC1 (a complex sensitive to rapamycin), is a kinase downstream of Akt (Yang and Guan, 2007; Huang and Manning, 2009) that is expressed in sensory fibers and dorsal horn neurons (Jiménez-Díaz et al., 2008; Géranton et al., 2009; Xu et al., 2010). Inhibition of spinal mTORC1 by rapamycin is anti-nociceptive in models of tissue injury (Price et al., 2007; Jiménez-Díaz et al., 2008; Asante et al., 2009; Géranton et al., 2009; Norsted Gregory et al., 2010). Although mTORC1mediated protein translation is considered a major mechanism underlying its effects, mTORC1 activity may also directly modulate neuronal excitability.

These findings indicate an important role for the Akt-mTOR cascade in spinal nociceptive processing. However, little is known about the role of these functional linkages of spinal Akt with mTORC1 in conditions of persistent pain induced by peripheral inflammation. In this study, we address several questions to define the role of this linkage in post-tissue injury hyperpathia. 
(1) Is there evidence for increased dorsal horn Akt and mTOR activity with peripheral inflammation? (2) If so, in which cell types does this activation occur? (3) Does spinal Akt act as an upstream signal for mTOR activation after peripheral inflammation? (4) Does blockade of spinal Akt or mTOR activity alleviate inflammation-induced hyperalgesia? (5) Finally, is AktmTOR involved in the signaling of neurokinin 1 receptor (NK1R)-positive dorsal horn neurons (many of which are believed to be nociceptive projection neurons and involved in central sensitization and dorsal horn excitability (Suzuki et al., 2002; Todd et al., 2002).

\section{Materials and Methods}

Subjects. Male Holtzman Sprague Dawley rats (250-350 g; Harlan) were housed individually after surgery and maintained in a light-controlled room (lights on from 7:00 A.M. to 7:00 P.M.) at a constant temperature of $22^{\circ} \mathrm{C}$ with access to food and water ad libitum. Surgical procedures and testing occurred during the light cycle. All experiments were performed in accordance with the Guide for the Care and Use of Laboratory Animals (National Institutes of Health Publication 85-23) and protocols were approved by the University of California, San Diego, Institutional Animal Care and Use Committee. Measures were taken to minimize the pain and discomfort of the experimental animals.

Intrathecal catheter implantation. Rats were implanted with an intrathecal catheter for drug delivery, as described previously (Yaksh and Rudy, 1976; Malkmus and Yaksh, 2004). In brief, rats were anesthetized by isoflurane. The atlanto-occipital membrane was exposed and an incision was made. A catheter (single lumen polyethylene, $8.5 \mathrm{~cm}$ in length, $0.20 \mathrm{~mm}$ inner diameter/0.36 mm outer diameter, BB31695-PE/08; Scientific Commodities) was inserted through the incision into the intrathecal space and advanced to the level of the L1-L2 spinal segments. The other end of the catheter was joined to a polyethylene-10 catheter and tunneled through the skin. The catheter was flushed with saline and plugged. The wounds were closed with 3-0 silk sutures. The rats were allowed to recover for at least $5 \mathrm{~d}$ before the experiment. Rats were monitored daily and removed from the study if any neurological dysfunction was noted, if there was $>10 \%$ weight loss over $5 \mathrm{~d}$, or if the catheters were occluded.

Drugs: preparation and administration. For intrathecal injection, all agents were prepared to be delivered in $10 \mu \mathrm{l}$, followed by $10 \mu \mathrm{l}$ of saline to flush the catheter. Substance P (SP) (30 nmol; Sigma) was dissolved in physiological saline $(0.9 \% \mathrm{NaCl})$. Wortmannin (Sigma), Akt-inhibitor$1 / 2$ (Akt-I-1/2), and PI-103 (Calbiochem) were dissolved in 3\% dimethylsulfoxide (DMSO) in physiological saline. Rapamycin (Calbiochem) was dissolved in 5\% ethanol and 10\% cyclodextran. Wortmannin was injected within $10 \mathrm{~min}$ after preparation.

Western blot. Rats were deeply anesthetized by isoflurane and decapitated, and the spinal cords were hydroextruded from the vertebral column using a $10 \mathrm{ml}$ syringe filled with ice-cold saline. The dorsal quadrants of the lumbar spinal cord were dissected and immediately snap-frozen on dry ice. Samples were homogenized in extraction buffer [50 mM Tris buffer, pH 7.4, containing 0.5\% Triton X-100, $150 \mathrm{~mm} \mathrm{NaCl}$, 1 mм EDTA, 3\% SDS, protease inhibitor cocktail (1:100, P8340; Sigma), and phosphatase inhibitor cocktail I and II (1:100, P2850 and P5726; Sigma) by sonication. The homogenized samples were centrifuged at $14,500 \mathrm{rpm}$ for $15 \mathrm{~min}$ at $4^{\circ} \mathrm{C}$. The supernatants were collected and aliquoted for storage at $-70^{\circ} \mathrm{C}$. The protein concentration for each sample was determined using a BCA protein assay kit (Pierce). Samples containing equal weights of total protein $(30 \mu \mathrm{g})$ were separated by denaturing and reducing SDS-PAGE using $4-12 \%$ Bis-Tris gel with 3-(N-morpholino)-propanesulfonic acid running buffer (Invitrogen). The separated proteins were then transferred to nitrocellulose membranes using Invitrogen iBlot dry blotting system.

All membrane incubations were performed on a rotating plate. Washes were performed in TBS- $\mathrm{T}$ ( $50 \mathrm{~mm}$ Tris- $\mathrm{Cl}$ and $6 \mathrm{~mm} \mathrm{NaCl}$ containing $0.1 \%$ Tween 20). Primary antibodies were diluted in TBS-T containing $5 \%$ bovine serum albumin, Fraction V (Sigma). Blocking of nonspecific binding sites was accomplished by incubating the mem-
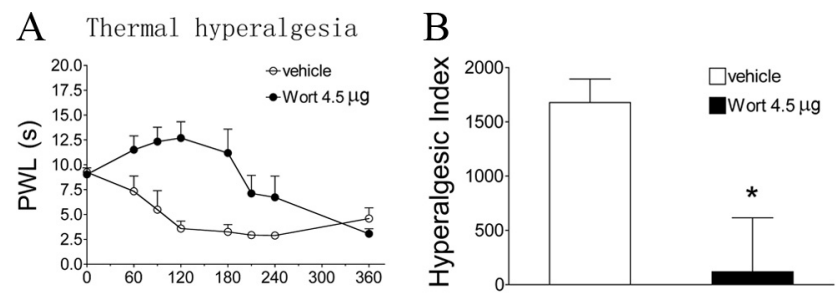

Time after Carrageenan (min)
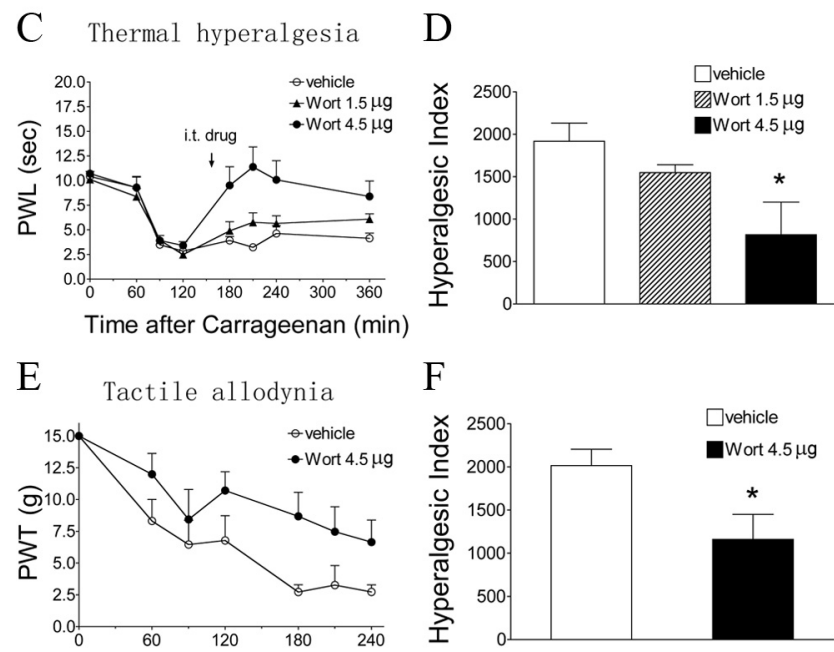

Time after Carrageenan $(\mathrm{min})$

Figure 1. Effects of intrathecal wortmannin on intraplantar carrageenan-induced pain behaviors. $A, C$, Time course over $360 \mathrm{~min}$ of PWL to thermal stimulation in the injected paw before (baseline, time 0 ) and after carrageenan. Intrathecal (IT) vehicle (3\% DMSO, $10 \mu \mathrm{l}$ ) or wortmannin (Wort; $4.5 \mu \mathrm{g}$ in $\boldsymbol{A} ; 1.5$ and $4.5 \mu \mathrm{g}$ in $\boldsymbol{B}$ ) was administered $15 \mathrm{~min}$ before $(\boldsymbol{A})$ or $130 \mathrm{~min}$ after $(\boldsymbol{C})$ intraplantar carrageenan $(2 \%, 100 \mu \mathrm{l}) . \boldsymbol{B}, \mathbf{D}$, Histograms represent the hyperalgesia index from 0 to $360 \mathrm{~min}$. $E$, Time course over $240 \mathrm{~min}$ of PWT to tactile stimulation in the injected paw before and after carrageenan. $\boldsymbol{F}$, Histogram represents the hyperalgesia index from 0 to 240 min intrathecal vehicle or wortmannin $(4.5 \mu \mathrm{g})$ was given $15 \mathrm{~min}$ before carrageenan. The data are presented as mean \pm SEM of six to eight rats per group. ${ }^{*} p<0.05$ compared with the vehicle by $t$ test $(\boldsymbol{B}, \boldsymbol{F})$ or one-way ANOVA (D).

branes in 5\% nonfat dry milk in TBS-T for $1 \mathrm{~h}$ at room temperature. The membranes were then incubated with the primary antibody overnight at $4^{\circ} \mathrm{C}$. After washing, membranes were probed with appropriate horseradish peroxidase-conjugated secondary antibody diluted at 1:10,000 with $5 \%$ nonfat dry milk in TBS-T for $1 \mathrm{~h}$ at room temperature. After washing, membranes were incubated according to the directions of the manufacturer with SuperSignal Chemiluminescent Substrate reagents (Pierce), and the luminescent signal was exposed to film and developed for additional scanning and quantification. The nitrocellulose membranes were stripped with a Re-Blot Western blot recycling kit (Millipore Bioscience Research Reagents) and reprobed with appropriate primary antibodies. Antibodies to pAkt (ser473, 1:1000; catalog \#9271), total Akt (1:1000; catalog \#9272), pmTOR (ser2448, 1:1000; catalog \#2971), total mTOR (1:1000; catalog \#2972), pS6 (ser235/236, 1:500; catalog \#2211), total S6 (1:500; catalog \#2217), p4EBP (thr37/46, 1:500; catalog \#9459), and total 4EBP (1:1000; catalog \#9452) were obtained from Cell Signaling Technology. $\beta$-Actin $(1: 50,000)$ were obtained from Sigma. The optical density of immunoreactive bands was quantified using ImageQuant software (Molecular Dynamics). All phospho bands were normalized relative to the corresponding $\beta$-actin band.

Immunohistochemistry. Animals were deeply anesthetized with Euthasol (0.5 ml; Virbac Animal Health) and transcardially perfused with 100 $\mathrm{ml}$ of $0.9 \% \mathrm{NaCl}$, followed by $200 \mathrm{ml}$ of $4 \%$ paraformaldehyde in $0.1 \mathrm{M}$ PBS. The lumbar spinal cords were dissected and postfixed in the same fixative for $2 \mathrm{~h}$ at $4^{\circ} \mathrm{C}$. The cords were then transferred to $30 \%$ sucrose in PBS and cryoprotected for $72 \mathrm{~h}$ at $4^{\circ} \mathrm{C}$. Thirty micrometer transverse sections were cut on a Leica cryostat (CM1800) and mounted on super- 
A
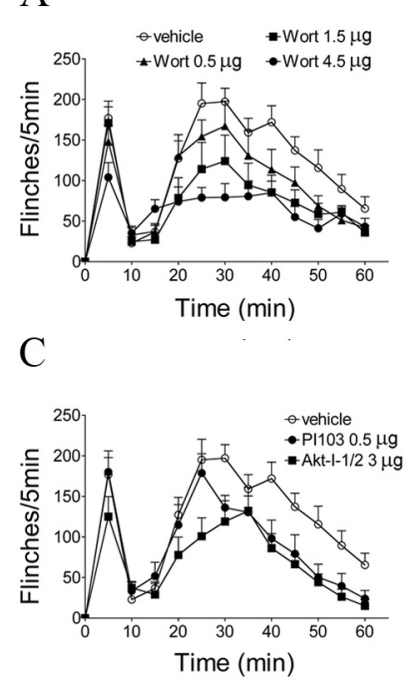

Figure 2. Effects of intrathecal injection of PI3K-Akt inhibitors on Formalin-induced flinching behaviors. $A, C$, Time course over $60 \mathrm{~min}$ of flinch responses after Formalin $(2.5 \%, 50 \mu \mathrm{l})$ paw injection in rats with intrathecal injection of vehicle (3\% DMSO, $10 \mu \mathrm{l}, 15 \mathrm{~min}$ before Formalin) or wortmannin (Wort; $0.5-4.5 \mu \mathrm{g})(\boldsymbol{A})$ or vehicle (3\% DMSO), PI-103 $(0.5 \mu \mathrm{g})$, or Akt-I-1/2 $(3 \mu \mathrm{g})(\boldsymbol{C})$. Each point represents the number of flinches in a 5 min block. B, D, Histograms represent the total number of flinches in each phase: phasel, 1-9 min; and phase ll, $10-60 \mathrm{~min}$. The data are presented as mean \pm SEM of six to eight rats per group. ${ }^{*} p<0.05$ compared with vehicle ( $3 \%$ DMSO) by one-way ANOVA, followed by Tukey's post hoc test.

frost plus slides (12-550-15; Thermo Fisher Scientific) before proceeding to immunohistochemistry (IHC). Nonspecific binding was blocked by incubation in 10\% normal goat serum (S1000; Vector Laboratories) in PBS with $0.3 \%$ Triton $\mathrm{X}-100$, followed by incubation a rabbit polyclonal antibody [Akt (ser473, 1:200; catalog \#4060; Cell Signaling Technology), pS6 (ser235/236, 1:500; catalog \#2211; Cell Signaling Technology), p4EBP (thr37/46, 1:500; antibody 1, catalog \#9459; antibody 2, catalog \#2855; Cell Signaling Technology) or pmTOR (ser2448, 1:200; catalog \#2971; Cell Signaling Technology)] and a mouse monoclonal antibody [neuronal specific nuclear protein (NeuN) (1:500; MAB377; Millipore Bioscience Research Reagents), glial fibrillary acidic protein (GFAP) (1: 1000; MAB360; Millipore Bioscience Research Reagents) or OX-42 (1: 1000; MCA275GA; AbD Serotec)] for $72 \mathrm{~h}$ at $4^{\circ} \mathrm{C}$. For double labeling of $\mathrm{NK} 1 \mathrm{R} / \mathrm{p}-\mathrm{Akt} / \mathrm{pmTOR} / \mathrm{pS} 6$, free-floating spinal sections $(60 \mu \mathrm{m})$ were first incubated with the first antibody $(1: 20,000$ to $1: 40,000)$ at $4^{\circ} \mathrm{C}$ for $72 \mathrm{~h}$. Sections were then incubated with biotinylated anti-rabbit serum followed by ABC complex (Vector Laboratories). Color was developed by incubating with cyanine 3-tyramide (PerkinElmer Life and Analytical Sciences). Sections were then washed and incubated with the respective second antibody (1:200 to 1:500) and Alexa Fluor 488 anti-rabbit antibody. All antibodies were diluted in SignalStain Antibody Diluent with $0.3 \%$ Triton X-100 (8112; Cell Signaling Technology). Coverslips were mounted with ProLong antifade medium (Invitrogen) containing TOPRO-3 (1:1000; T3605; Invitrogen). Nonspecific labeling was determined by excluding the primary antibodies.

Confocal microscopy and image processing. Confocal images were acquired by a Leica TCS SP5 confocal system using sequential scanning to avoid fluorescence bleed-through. Images were acquired at a digital size of $1024 \times 1024$ pixels. To demonstrate changes in the dorsal horn following intraplantar carrageenan, 10 adjacent optical sections (20 $\mu \mathrm{m}$ depth) along the $z$-axis were projected together. The projected images were then adjusted with Adobe Photoshop software to enhance image clarity.

Cell counting. pS6-labeled neurons were counted in three domains in the ipsilateral spinal dorsal horn and laminae I/II, III/IV, and V/VI. Three random spinal sections from the L4-L5 region were counted for each rat. Four rats were included in each treatment group.

Formalin-induced flinching behavior. An automated system was used to quantify Formalin-induced flinching (University Anesthesia Research
A
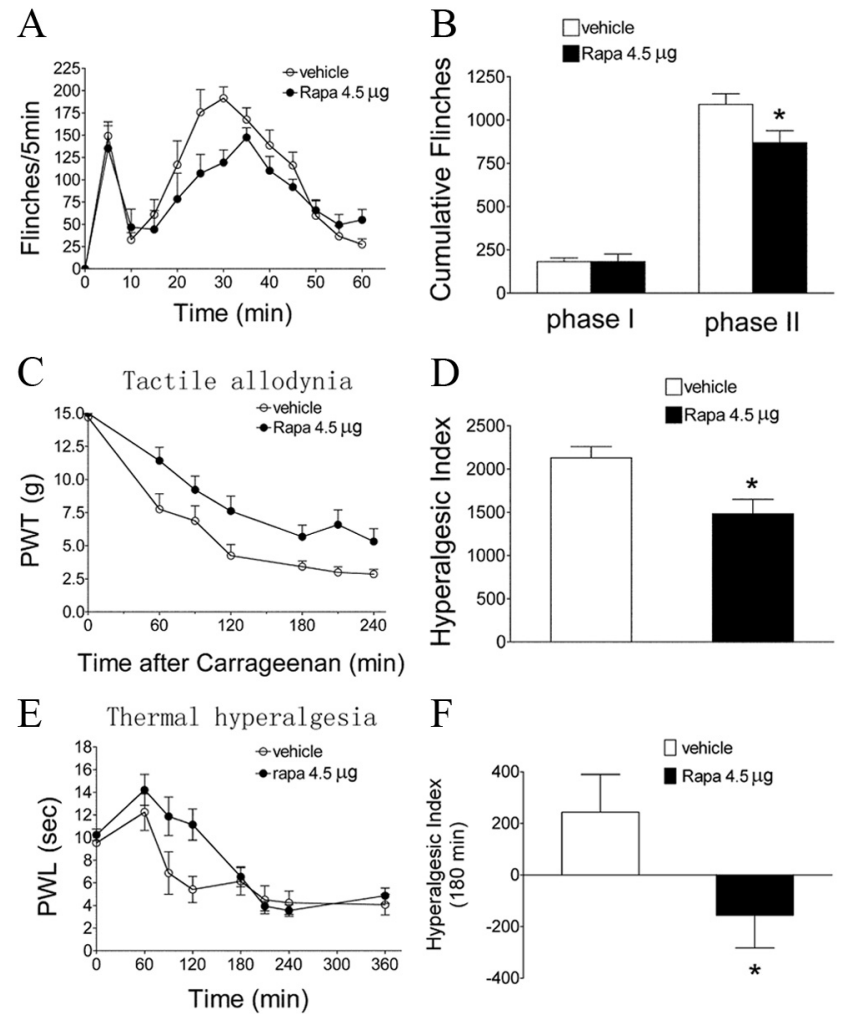

Figure 3. Effects of intrathecal rapamycin on Formalin or carrageenan-induced pain behaviors. $\boldsymbol{A}$, Time course over $60 \mathrm{~min}$ of flinch responses after Formalin $(2.5 \%, 50 \mu \mathrm{l})$. Each point represents the number of flinches in a 5 min block. $\boldsymbol{B}$, Histogram represents the total number of flinches in each phase: phase I, 1-9 min; and phase II, $10-60 \mathrm{~min}$. C, E, Time course of PWT to tactile stimulation $(\boldsymbol{C})$ and PWL to thermal stimulation $(\boldsymbol{E})$ in the injected paw before (baseline, time 0 ) and after carrageenan $(2 \%, 100 \mu \mathrm{l}) . \boldsymbol{D}, \boldsymbol{F}$, Histograms represent the hyperalgesia index over the testing period ( $240 \mathrm{~min}$ in $\boldsymbol{D}$ and $180 \mathrm{~min}$ in $\boldsymbol{F}$ ). Intrathecal vehicle (3\% DMSO) or rapamycin (Rapa; $4.5 \mu \mathrm{g}$ ) was administered $15 \mathrm{~min}$ before Formalin or carrageenan. The data are presented as mean \pm SEM of six to eight rats per group. ${ }^{*} p<0.05$ compared with the vehicle by $t$ test.

and Development Group, Department of Anesthesiology, University of California, San Diego, La Jolla, CA). Briefly, a soft metal band was placed around the left hindpaw of the animal. After acclimation, rats were dosed intrathecally with wortmannin $(0.5,1.5$, or $4.5 \mu \mathrm{g})$, PI-103 $(1.5 \mu \mathrm{g})$, Akt-I-1/2 $(0.5 \mu \mathrm{g})$, rapamycin $(4.5 \mu \mathrm{g})$, or vehicle. Fifteen minutes after intrathecal drug administration, the dorsal side of left paw was injected with Formalin solution (2.5\% in physiological saline, $50 \mu \mathrm{l})$ and placed into the test chamber immediately. Nociceptive behavior was quantified by automatic counting of the incidence of flinching and shaking of the injected paw. Flinches were counted in $1 \mathrm{~min}$ intervals for $60 \mathrm{~min}$. The data are expressed as number of flinches per $5 \mathrm{~min}$ over the $60 \mathrm{~min}$ period and total number of flinches observed during phase I (0-9 min) and phase II (10-60 min). Rats were killed after each behavior testing session (Yaksh et al., 2001).

Intraplantar carrageenan-induced thermal hyperalgesia and mechanical allodynia. All behavioral measurements were made by observers blinded to the treatment groups. Peripheral inflammation was produced by injecting $\lambda$-carrageenan (100 $\mu \mathrm{l}, 2 \%$ in saline; Nacalai Tesque) into the plantar surface of one hindpaw under a brief isoflurane anesthesia (2$4 \%)$. To assess the thermally evoked paw-withdrawal response, a Hargreaves-type device was used (Dirig et al., 1997).

Briefly, in this procedure, rats are acclimated in the test chamber for $30 \mathrm{~min}$ on a glass surface maintained at $25^{\circ} \mathrm{C}$. The radiant heat generated by a halogen projection bulb is used to stimulate the hindpaw. An abrupt withdrawal of the paw is detected by a motion sensor, which triggers the termination of the stimulus and paw-withdrawal latency (PWL) is determined automatically. A cutoff of $20 \mathrm{~s}$ is used to avoid tissue injury. 
Withdrawal latencies in response to thermal stimuli were measured before carrageenan (baseline) and at times 60, 90,120, 180, 210, 240, and 360 min after carrageenan. Pawwithdrawal thresholds (PWTs) in response to innocuous mechanical stimuli were measured before carrageenan (baseline) or at times 60, $90,120,180,210$, and 240 min after carrageenan. PWTs were determined by application of a series of calibrated von Frey filaments to the surface of the hindpaws (Chaplan et al., 1994). The animals were acclimated for $30 \mathrm{~min}$ in the test chamber on mesh flooring suspended above the observer, and von Frey filaments were applied perpendicularly against the plantar surface of the paw. The "up-down" method of Dixon was used to determine the value at which paw withdrawal occurred $50 \%$ of the time, interpreted as the mechanical threshold.

Intrathecal SP-induced hyperalgesia. Intrathecal SP-induced thermal hyperalgesia is a model in which the animals have no peripheral injury and the hyperalgesia is initiated by a direct activation of spinal NK1Rs (Piercey et al., 1981; Yashpal and Henry, 1983). Paw-withdrawal latencies in response to thermal stimuli were measured before intrathecal injection of SP at $30 \mathrm{nmol}$ (baseline) and at times 10, 20, 30, and $60 \mathrm{~min}$ after SP using a Hargreaves-type device (see above).

Statistics. The behavioral data of PWL or PWT are presented as the mean \pm SEM at each time point and are also expressed as the hyperalgesic index, a calculation that defines the magnitude of hyperalgesia. The hyperalgesic index represents the area under the time effect curve after stimulation in which the "percent reduction from baseline (e.g., pre-carrageenan) response latency" is plotted against time. The resulting metric is percentage change $\times$ minutes. The formula for calculating the percentage change is (baseline latency - post-drug latency) $\times 100$ (baseline latency) ${ }^{-1}$, where latency is expressed in seconds per grams. Increasing values reflect increasing hyperalgesia.

For Western blotting and behavior data, one-way ANOVA followed by a Tukey's post hoc test was used when more than two groups of data were compared; Student's $t$ test was used when two groups were compared.

\section{Results \\ Inhibition of spinal PI3K-Akt-mTOR pathway attenuates inflammatory hyperalgesia}

To define involvement of spinal PI3K-Akt and mTOR in inflammation-induced hyperalgesia, we examined the effects of intrathecal delivery of inhibitors of individual kinases in two behavioral models, intraplantar carrageenan-induced thermal hyperalgesia and tactile allodynia, and Formalin-induced flinching behavior.

Baseline PWL to a thermal stimulus in the vehicle-treated group was $9.2 \pm 0.4 \mathrm{~s}$ (Fig. $1 \mathrm{~A}$ ). Intraplantar carrageenan produced an ipsilateral reduction in the thermal latency to $3.6 \pm 0.8 \mathrm{~s}$ $(p<0.05)$ at $2 \mathrm{~h}$, an effect that lasted for at least $4 \mathrm{~h}$. Wortmannin administered intrathecally $(4.5 \mu \mathrm{g})$ before carrageenan resulted in a potent antihyperalgesic effect (Fig. $1 A, B)$. Wortmannin (1.5 or $4.5 \mu \mathrm{g}$ ) given intrathecally in animals with an established thermal hyperalgesia resulted in a significant elevation of the decreased latencies $(p<0.05)$ compared with vehicle (Fig. 1C,D). no $\operatorname{carr}$
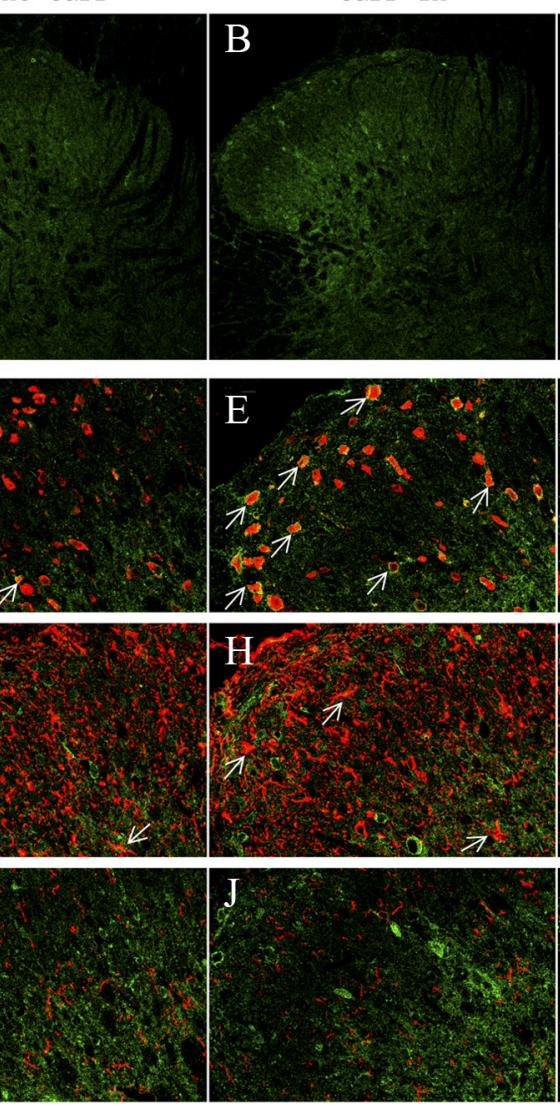

$\operatorname{carr} 4 \mathrm{~h}$
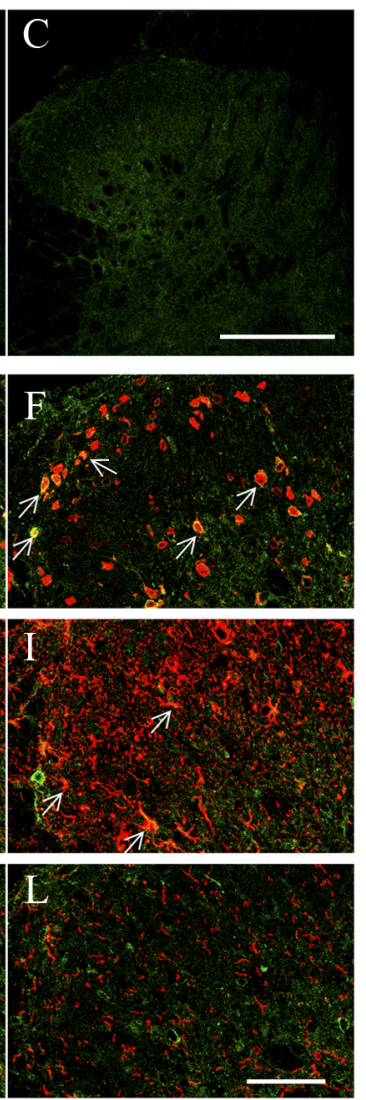

Figure 4. Distribution and cellular localization of pAkt immunoreactivity in the ipsilateral spinal cord dorsal horn after in(l) compared with naive. A pronounced increase is seen in laminae I, III, and IV. Ten confocal planes along the z-axis plane images of pAkt (green) with NeuN (D-F, red), GFAP $(\mathbf{G}-\boldsymbol{I}$, red), or 0X42 (J-L, red) are shown. Arrows indicate colocalization of pAkt with the respective cell markers (yellow). Scale bars: $A-C, 250 \mu \mathrm{m} ; \boldsymbol{D}-\boldsymbol{L}, 50 \mu \mathrm{m}$.

In animals pretreated with intrathecal vehicle, mechanical thresholds were decreased after carrageenan (from baseline $15 \mathrm{~g}$ down to $3.4 \pm 0.4 \mathrm{~g}$ at $3 \mathrm{~h}, p<0.05)$. Pretreatment with intrathecal wortmannin $(4.5 \mu \mathrm{g})$ attenuated carrageenan-induced allodynia (Fig. $1 E, F, p<0.05$ ). In these behavioral tests, intrathecal wortmannin did not alter the thermal latencies or mechanical thresholds of the contralateral (un-inflamed) paws (data not shown).

Formalin injection into the hindpaw resulted in a characteristic biphasic flinching behavior, the second phase of which (10-60 min) is considered to represent spinal sensitization (Puig and Sorkin, 1996). Intrathecal pretreatment with wortmannin produced a dosedependent (0.5-4.5 $\mu \mathrm{g}$ ) inhibition of phase II flinching (Fig. $2 A, B$, $p<0.05)$ compared with vehicle treatment. In addition, we tested the effect of two other inhibitors on Formalin-induced flinches. Pretreatment with intrathecal PI-103 $(0.5 \mu \mathrm{g})$, an inhibitor of PI3K, or Akt-I-1/2 $(3 \mu \mathrm{g})$, an inhibitor of Akt, significantly reduced Formalin-induced phase II flinching (Fig. $2 C, D, p<0.05$ ). None of these inhibitors affected phase I behavior. At the doses examined, the observed effects of these agents were not accompanied by signs of sedation, motor weakness, or impaired ambulation.

To define the involvement of spinal mTOR, rapamycin, a selective inhibitor of mTORC1, was given intrathecally $15 \mathrm{~min}$ before Formalin. Rapamycin produced a significant inhibition of Formalin-induced phase II flinches and carrageenan-induced 
A

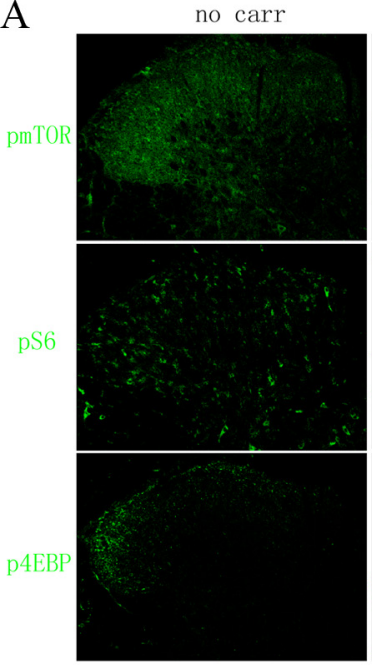

B

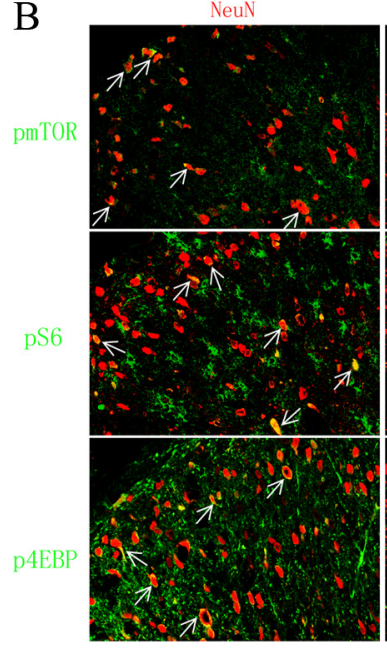

C

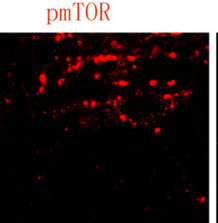

pmTOR

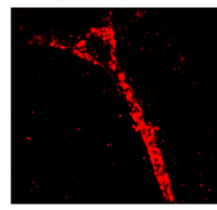

pAkt

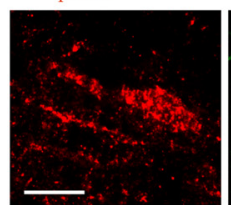

carr 1h

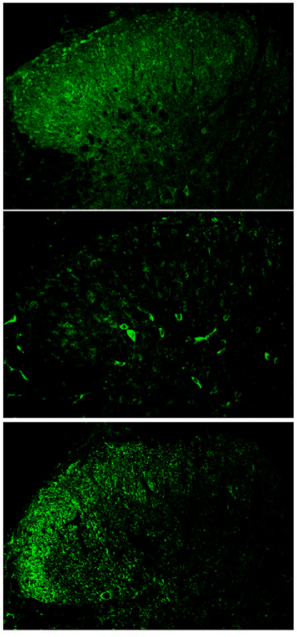

GFAP

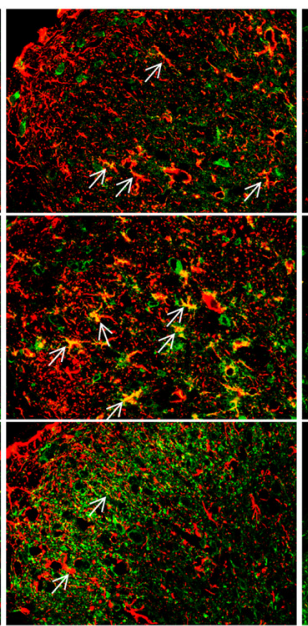

TO-PRO-3

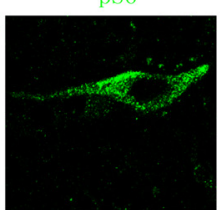

pAkt

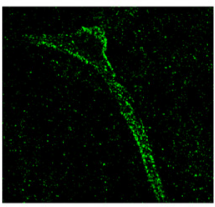

pS6

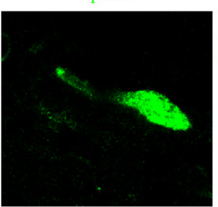

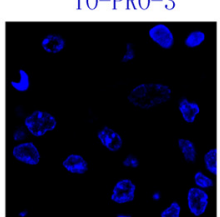

TO-PRO-3

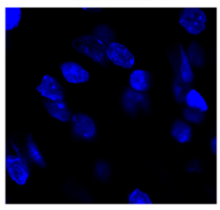

T0-PRO-3

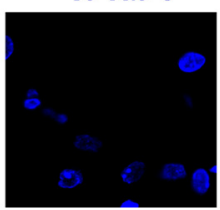

carr 4 h

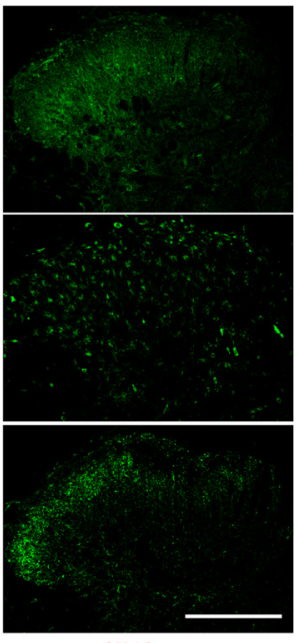

0X42
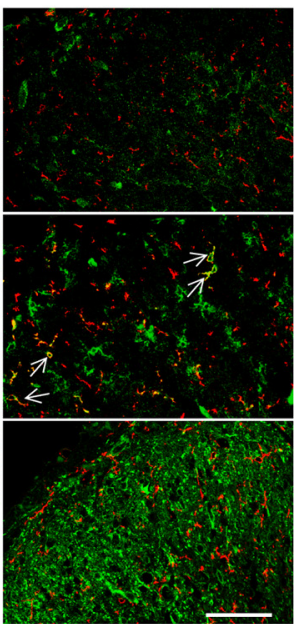

Merged

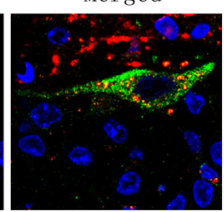

Merged

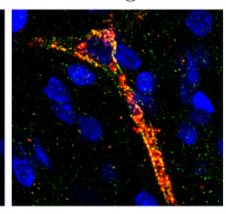

Merged

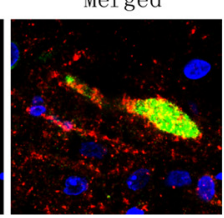

Figure 5. Distribution and cellular localization of pmTOR, pS6, p4EBP, and pAkt immunoreactivity in the ipsilateral spinal cord dorsal horn after intraplantar carrageenan. $\boldsymbol{A}$, Immunoreactivity of pmTOR, pS6, and p4EBP in the ipsilateral dorsal horn. Ten confocal planes along the $z$-axis are projected (20 $\mu \mathrm{m}$ depth). $\boldsymbol{B}$, (ell-type-specific immunolabeling of pmTOR, pS6, and p4EBP in the ipsilateral dorsal horn. Single focal plane images are shown. Arrows indicate colocalization of the phospho-proteins with the respective cell markers (yellow). C, Double labeling of pAkt, pmTOR, and pS6 in dorsal horn neurons at $1 \mathrm{~h}$ after intraplantar carrageenan. Sections are counterstained with T0-PRO-3 (blue) to label cell nuclei. Single focal plane images are shown. Scale bars: A, $250 \mu \mathrm{m} ; \boldsymbol{B}, 50 \mu \mathrm{m} ; \boldsymbol{C}, 20 \mu \mathrm{m}$.

tactile allodynia (Fig. $3 A-D, p<0.05$ ). In addition, rapamycin attenuated thermal hyperalgesia after carrageenan (Fig. $3 E, F$, $p<0.05$ ), although this effect seems to be shorter lasting (180 min) than its effect on tactile allodynia.

\section{Peripheral injury and inflammation- induced activation of Akt and mTOR in spinal dorsal horn}

In parallel with the time course of the development of hyperalgesia, the injection of carrageenan induced an increase in Akt phosphorylation (ser473) in the spinal cord dorsal horn ipsilateral to the injected paw (Fig. $4 A-C$ ), suggesting that Akt is activated in response to peripheral inflammation. The increase was most prominent at $1 \mathrm{~h}$ after carrageenan, in laminae I, III, and IV, compared with the naive tissue. Both punctate and diffuse labeling was visible, mostly in the gray matter, although some labeling was also seen in white matter. Confocal images demonstrated that the majority of pAkt-positive profiles were colocalized with the neuronal marker NeuN in the dorsal horn (Fig. $4 D-F)$, suggesting that inflammationtriggered activation of Akt occur primarily in dorsal horn neurons. pAkt was noted in only a few GFAP-positive cells (astrocytes) and almost none in OX42positive (microglia) cells (Fig. 4G-L).

Similar to pAkt, a marked increase in pmTOR (ser2448), pS6 (ser235/236), and p4EBP (thr37/46) was observed in the ipsilateral laminae I, III, and IV, most prominently at $1 \mathrm{~h}$ after carrageenan (Fig. 5A). Both pmTOR and pS6 colabeled with NeuN and GFAP (Fig. 5B), whereas pS6 was also detected in some OX42-positive profiles (Fig. 5B). Immunocytochemical examination of expression and cellular localization of p4EBP was performed by using two individual antibodies from Cell Signaling Technology (antibody 1, catalog \#9459; antibody 2, catalog \#2855). The immunolabeling by both antibodies revealed that $\mathrm{p} 4 \mathrm{EBP}$ was increased in the superficial dorsal horn $1 \mathrm{~h}$ after intraplantar carrageenan (Figs. 5A, 6A). However, the pattern of cellular distribution was different. Antibody 1 showed colabeling of p4EBP with NeuN and GFAP (Fig. 5B), which is consistent with a previous report using the same antibody (Géranton et al., 2009), whereas antibody 2 showed a p4EBP colabeling with GFAP and OX42 but not NeuN (Fig. 6B). Based on the data obtained with both antibodies, we confirmed that $\mathrm{p} 4 \mathrm{EBP}$ is expressed in astrocytes. We do not know, however, whether 4EBP isoforms are presented in a cell- 
type-specific manner and whether the two antibodies preferably bind to different isoforms of phosphorylated 4EBP.

An important observation is shown in Figure $5 C$, in which colabeling of pAkt-pmTOR, pAkt-pS6, and pmTOR-pS6 is in dorsal horn neurons after intraplantar carrageenan injection. This provides morphological evidence to link Akt to mTOR signaling pathways after peripheral inflammation and confirms that activation of Akt and mTOR occurs in the same dorsal horn neuron.

Consistent with the immunohistochemistry data, Western blots revealed an increase in pAkt and pmTOR in the ipsilateral lumbar dorsal horn after intraplantar carrageenan (Fig. 7A-D). There was a significant increase in pAkt in dorsal cord after carrageenan administration, which peaked at $1 \mathrm{~h}$ (Fig. $7 A, B, p<0.05$ ) and remained elevated at $4 \mathrm{~h}$. The appearance of pmTOR follows a similar temporal pattern as that for pAkt, i.e., a profound increase was seen at $1 \mathrm{~h}$ (Fig. $7 C, p<0.05$ ), and these levels remained elevated at $4 \mathrm{~h}$ after carrageenan. Injection of Formalin into the hindpaw evoked a transient increase in Akt phosphorylation in the ipsilateral dorsal quadrant at $30 \mathrm{~min}$ after Formalin (Fig. $8 A, B, p<0.05$ ). The level of pAkt returned to the baseline by $1 \mathrm{~h}$ (data not shown). We did not detect any significant increase in pmTOR levels after Formalin (Fig. 8C,D). However, the analysis of IHC images revealed a significant increase of neurons labeled with pS6 ribosomal protein, a marker for mTORC1 activation, in the ipsilateral spinal cord dorsal horn (Fig. 8E,F).

To determine whether the activation of Akt and mTORC1 signaling produced by Formalin and carrageenan injections were mediated though spinal PI3K, we examined the effect of intrathecal wortmannin at dose of $4.5 \mu \mathrm{g}$, which was anti-hyperalgesic on the pain behavioral tests. This dose significantly attenuated Formalin or carrageenanevoked increases in pAkt as revealed by Western blot (Fig. 9A-E). In addition, intrathecal wortmannin blocked the phosphorylation of S6 induced by carrageenan (Fig. $10 A-D)$, suggesting that mTOR activation occurs downstream of PI3K-Akt signaling. Western blot also showed that the increase of pS6 was reduced by intrathecal rapamycin (Fig. 10E,F).

Spinal PI3K-Akt-mTOR is involved in NK1R-mediated pain signaling

Because both pAkt and pmTOR were increased in superficial dorsal horn neurons after peripheral inflammation, we sought to determine whether the facilitatory ef-
A
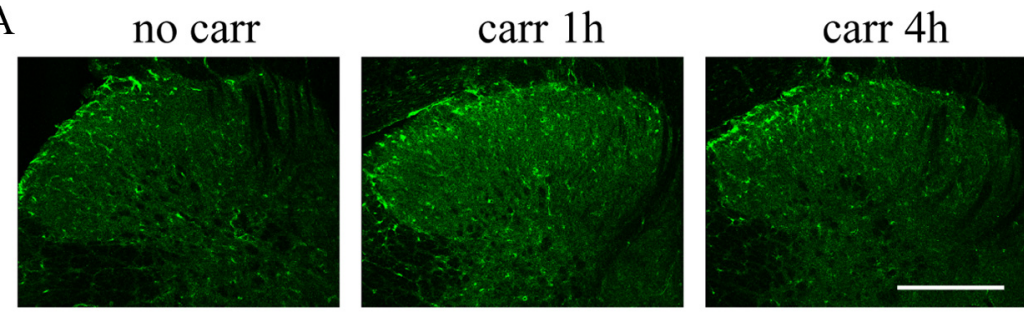

B

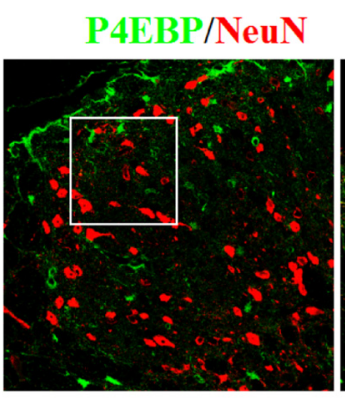

p4EBP/GFAP

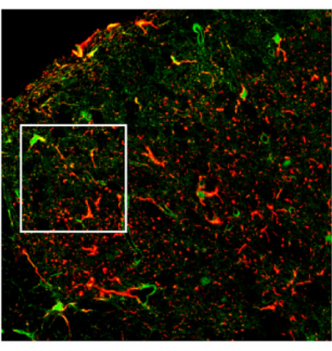

p4EBP/OX42
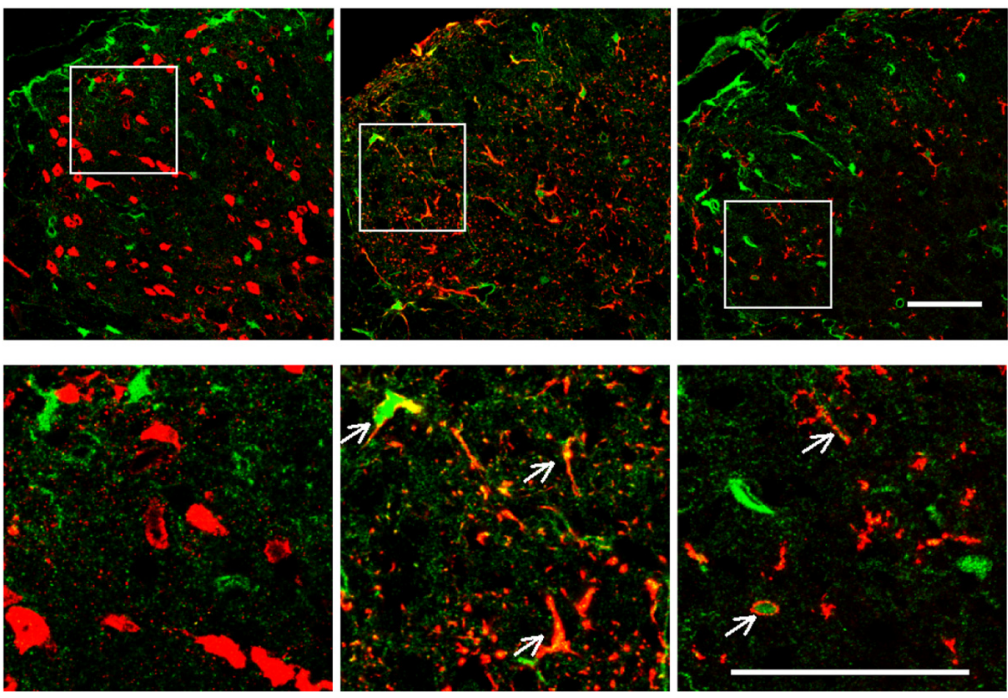

Figure 6. Distribution and cellular localization of p4EBP (catalog \#2855; Cell Signaling Technology) immunoreactivity in the ipsilateral spinal cord dorsal horn after intraplantar carrageenan. $A$, Immunoreactivity of $\mathrm{p4EBP}$ in the ipsilateral dorsal horn. Ten confocal planes along the $z$-axis are projected ( $20 \mu \mathrm{m}$ depth). $\boldsymbol{B}$, Cell-type-specific immunolabeling of p4EBP in the ipsilateral dorsal horn at $1 \mathrm{~h}$ after intraplantar carrageenan. Single focal plane images are shown. Arrows indicate colocalization of the phospho-proteins with the respective cell markers (yellow). Scale bars: $A, 250 \mu \mathrm{m} ; \boldsymbol{B}, 50 \mu \mathrm{m}$.
A

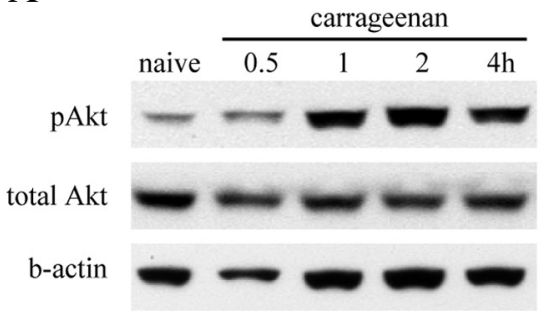

$\mathrm{C}$

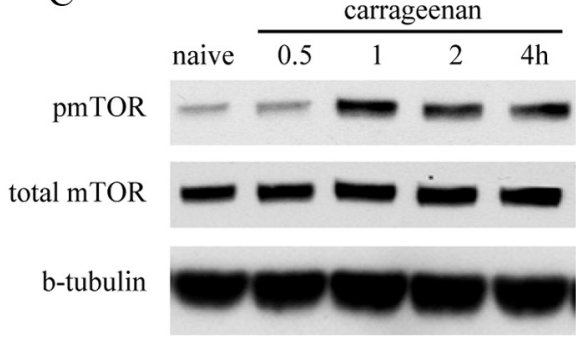

B

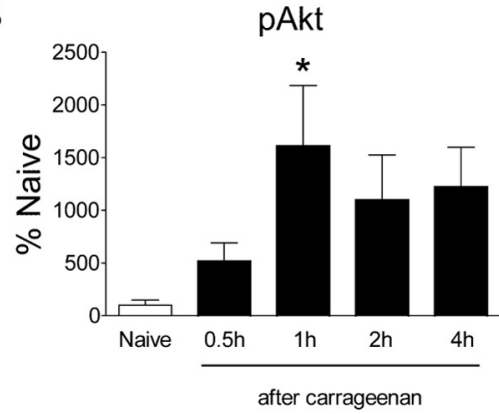

$\mathrm{D}$

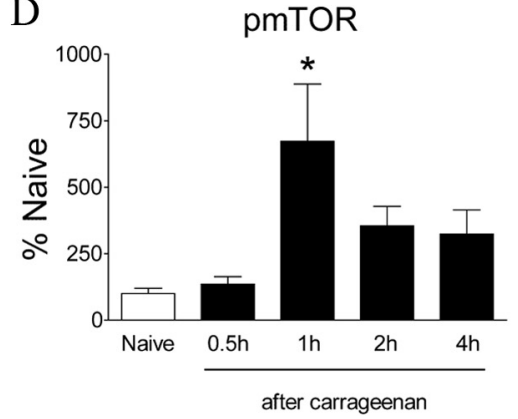

Figure 7. Carrageenan-induced changes in spinal levels of pAkt and pmTOR assessed by Western blot. $A, C$, Representative Western blots showing levels of pAkt, total Akt, and $\beta$-actin (b-actin) $(A)$ and pmTOR, total mTOR, and $\beta$-tubulin $(C)$ in the ipsilateral lumbar spinal dorsal quadrant at different time points after intraplantar carrageenan. $\boldsymbol{B}, \boldsymbol{D}$, Histograms represent the mean phosphor-protein levels with respect to the naive group. The data are presented as mean \pm SEM of three to five rats per group. ${ }^{*} p<0.05$ compared with naive by one-way ANOVA, followed by Tukey's post hoc test. 
A

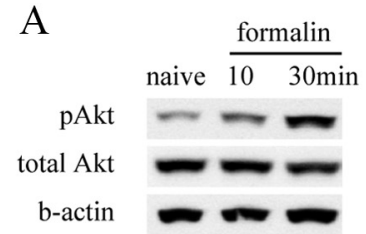

$\mathrm{C}$

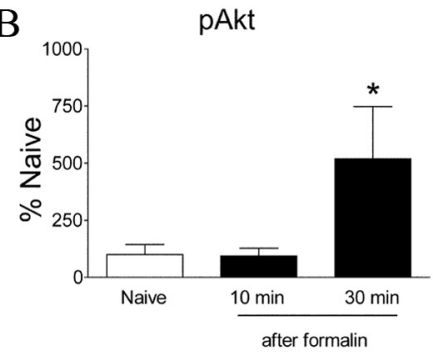

$\mathrm{D}$

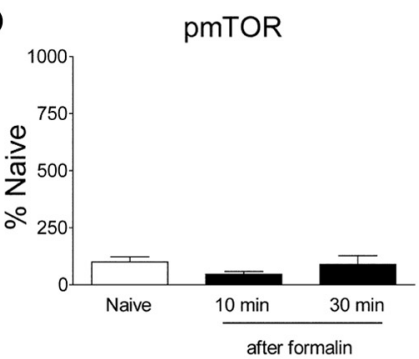

$\mathrm{E}$

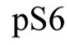

pS6

\section{contralateral}
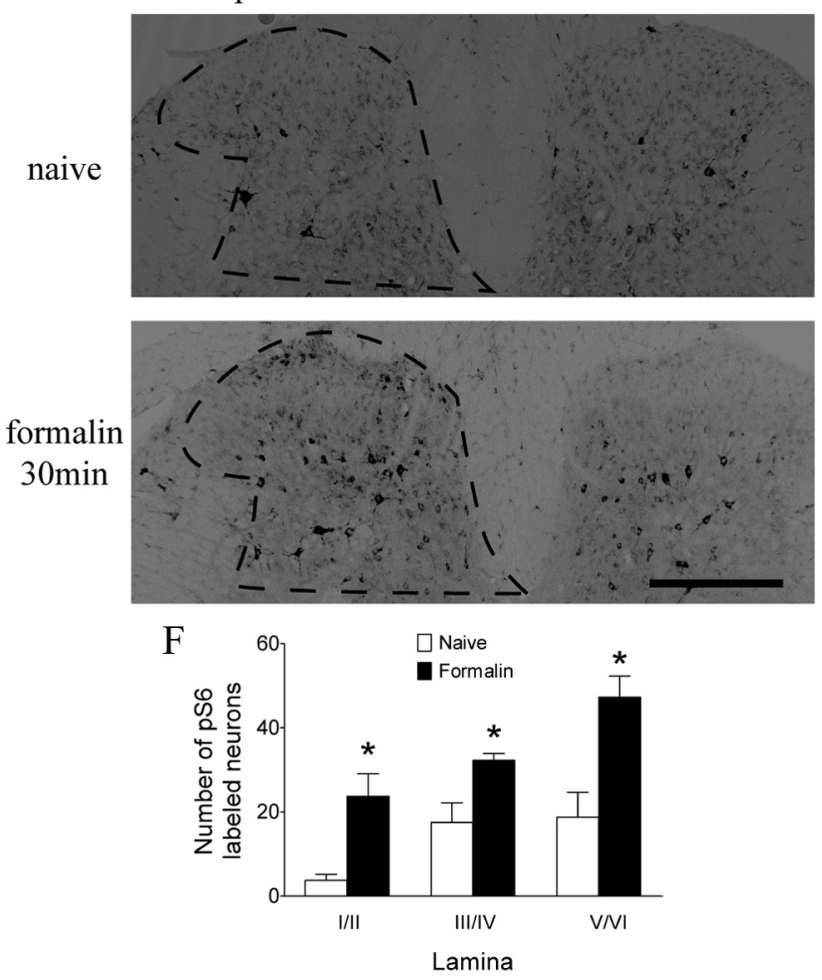

Figure 8. Formalin-induced changes in spinal levels of pAkt, pmTOR, and pS6 ribosomal protein. $A, C$, Representative Western blots showing levels of pAkt, total Akt, and $\beta$-actin (b-actin) $(\boldsymbol{A})$ and pmTOR, total mTOR, and $\beta$-tubulin $(\boldsymbol{C})$ in the ipsilateral lumbar spinal dorsal quadrant at different time points after Formalin. $\boldsymbol{B}, \boldsymbol{D}$, Histograms represent the mean pAkt or pmTOR level with respect to the naive group. $\boldsymbol{E}$, Immunoreactivity of p 56 ribosomal protein in the spinal dorsal horn in naive and Formalin-injected animals. $\boldsymbol{F}$, Histogram represents number of neurons labeled with pS6 in laminae I/II, III/IV, and V/VI. The data are presented as mean \pm SEM of three to five rats per group. ${ }^{*} p<0.05$ compared with naive by one-way ANOVA, followed by Tukey's post hoc test $(\boldsymbol{B}, \boldsymbol{D})$ or $t$ test $(\boldsymbol{F})$.

fect of this spinal PI3K-Akt-mTOR cascade indeed reflects an affiliation with dorsal horn circuits believed to convey nociceptive signals. NK1R-bearing neurons in laminae I and III/IV have synaptic contact with SP-containing small primary afferents (Todd et al., 2002). Many of these NK1R-positive cells are known to be projection neurons and to play a key role in spinal sensiti-
A

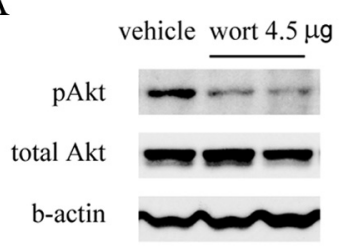

C
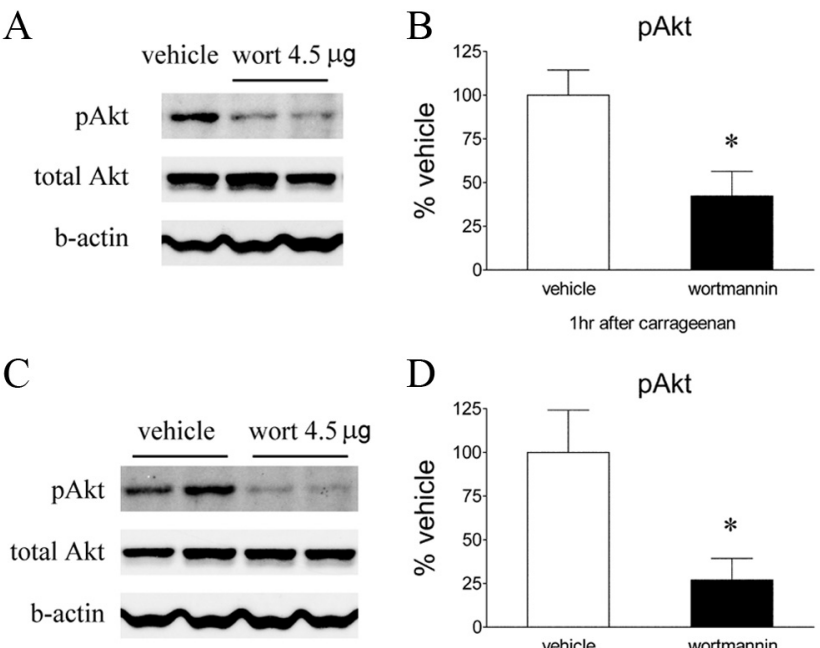

$\mathrm{D}$

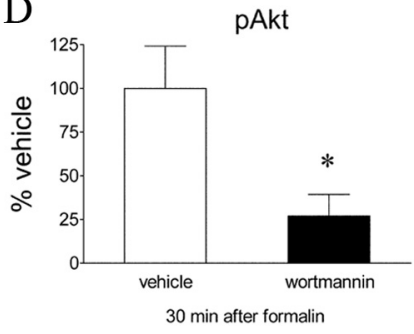

E

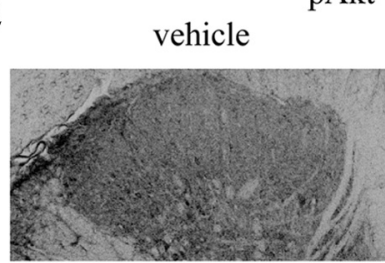

pAkt - carr $1 \mathrm{~h}$

Figure 9. Effects of intrathecal wortmannin on intraplantar carrageenan or Formalininduced pAkt. $\boldsymbol{A}, \boldsymbol{C}$, Representative Western blots showing pAkt, total Akt, and $\beta$-actin (b-actin) in the ipsilateral lumbar spinal dorsal quadrant at $1 \mathrm{~h}$ after carrageenan $(\boldsymbol{A})$ or $30 \mathrm{~min}$ after Formalin (C). Rats received intrathecal vehicle (3\% DMSO, $10 \mu \mathrm{l}$ ) or wortmannin (wort; $4.5 \mu \mathrm{g}$ ) $15 \mathrm{~min}$ before carrageenan or Formalin. $\boldsymbol{B}, \boldsymbol{D}$, Histograms represent the mean pAkt level with respect to the vehicle group. The data are presented as mean \pm SEM of four to eight rats per group. ${ }^{*} p<0.05$ compared with vehicle by $t$ test. $\boldsymbol{E}$, Immunoreactivity pAkt was reduced by intrathecal wortmannin compared with vehicle in dorsal horn at $1 \mathrm{~h}$ after intraplantar carrageenan. Scale bar, $250 \mu \mathrm{m}$.

zation though a spino-bulbo-spinal loop (Suzuki et al., 2002, 2005). Thus, it has been shown that abolition of NK1-bearing cells in superficial dorsal horn by intrathecal SP-saporin produces powerful anti-hyperalgesic effects in several models of inflammation and nerve injury (Nichols et al., 1999; Khasabov et al., 2002; Suzuki et al., 2002; Vierck et al., 2003). First, we showed that intrathecal SP $(30 \mathrm{nmol}, 30 \mathrm{~min})$ produced a profound increase of NK1R internalization in those NK1Rpositive cells (indicating their activation), and the internalized NK1R immunoreactivity colabeled with strong pAkt, pmTOR, or pS6 immunoreactivity (Fig. $11 \mathrm{~B}$ ). Second, we demonstrated that intrathecal SP at the same dose produced an expected thermal hypersensitivity (Fig. 12A,C), and this was prevented by pretreatment with intrathecal wortmannin or rapamycin (Fig. $12 A-D)$. Third, Western blots revealed a significant increase of pAkt and pS6 30 min after intrathecal SP in dorsal spinal cord compared with vehicle (Fig. $13 A, B, G, H$ ). Intrathecal wortmannin not only blocked the increase of pAkt but also the increase of pS6 (Fig. 13C-F), suggesting a sequential activation of Akt-mTORC1 that was triggered by intrathecal SP. Furthermore, intrathecal rapamycin blocked the SP-induced pS6 increase (Fig. $13 G, H)$. Fourth, we also observed in spinal cord tissues obtained from the rats that received intraplantar carrageenan $(1 \mathrm{~h})$ that pAkt and pmTOR immunoreactivities were expressed in NK1Rpositive neurons in laminae I, III, and IV in both soma and dendrites (Fig. 11 A). In combination with our findings that (1) both 
A

carrageenan $1 \mathrm{hr}$ vehicle wort $4.5 \mu \mathrm{g}$

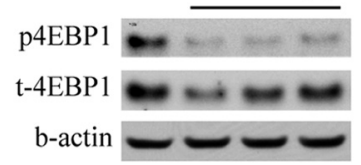

$\mathrm{C}$

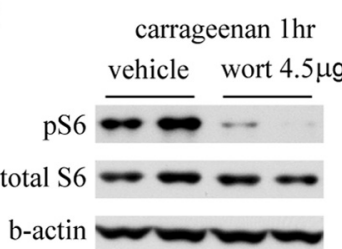

$\mathrm{E}$

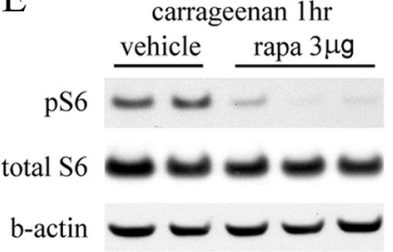

B

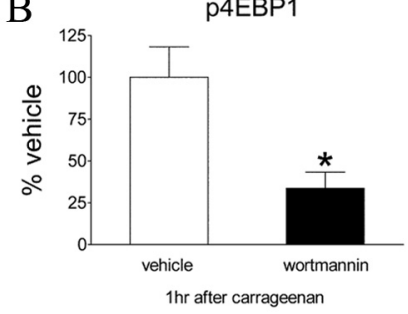

D

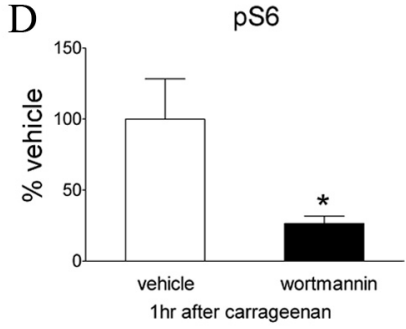

F

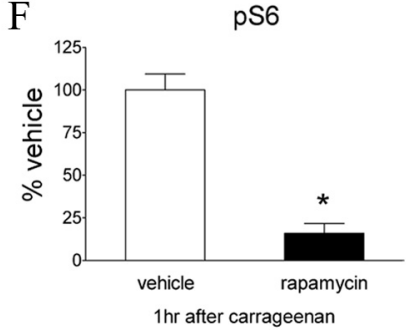

Figure 10. Effects of intrathecal wortmannin or rapamycin on intraplantar carrageenaninduced phosphorylation of $4 E B P$ and $S 6$ ribosomal protein. $A, C$, Representative Western blots showing p4EBP, total $4 E B P$, and $\beta$-actin ( $b$-actin) $(\boldsymbol{A})$ and pS6, total $S 6$, and $\beta$-actin $(\boldsymbol{C})$ in the ipsilateral lumbar spinal dorsal quadrant at $1 \mathrm{~h}$ after carrageenan. Rats received intrathecal vehicle (3\% DMSO, $10 \mu \mathrm{l}$ ) or wortmannin (wort; $4.5 \mu \mathrm{g}) 15$ min before carrageenan. $\boldsymbol{E}$, Western blots showing $\mathrm{pS} 6$, total $\mathrm{S6}$, and $\beta$-actin in rats received intrathecal vehicle (3\% DMSO, 10 $\mu$ l) or rapamycin (rapa; $3 \mu \mathrm{g}$ ) before carrageenan. $\boldsymbol{B}, \boldsymbol{D}, \boldsymbol{F}$, Histograms represent the mean $\mathrm{p} 4 \mathrm{EBP}$ and $\mathrm{pS} 6$ levels with respect to the vehicle group. The data are presented as mean \pm SEM of four or five rats per group. ${ }^{*} p<0.05$ compared with vehicle by $t$ test.

pAkt and pmTOR were increased significantly in dorsal horn after carrageenan at the same time point revealed by Western blotting, and (2) intrathecal SP produced expression of pAkt and pmTOR in the NK1R neurons, which also displayed NK1R internalization as revealed by IHC, we hypothesize that Akt and mTOR are likely activated in NK1R neurons in response to noxious input during inflammatory state.

\section{Discussion}

\section{Activation of the dorsal horn PI3K-Akt-mTOR cascade}

Within an hour of the onset of plantar inflammation and coincident with the appearance of spinal sensitization, there were increases in spinal expression of phosphorylated Akt and mTOR and in proteins downstream to mTOR (pS6 and p4EBP). The direct linkage between elements of this spinal cascade was demonstrated by blockade of spinal phosphorylation downstream of the respective drug target with intrathecal agents targeting PI3K, Akt, and mTOR. Immunohistochemistry indicates that the protein phosphorylation defined by Western blots is primarily associated with laminae I and III/IV neurons. The dense immunoreactivity of pAkt and pmTOR was observed in the lateral and medial aspects of the dorsal horn. This distribution corresponds with the input from the superficial peroneal, posterior cutaneous, saphenous, and sural nerves, which innervate, respectively, the dorsum, lower calf, medial aspect of the ankle, and the lateral aspect of the ankle (Swett and Woolf, 1985). This observation

A

carr $1 \mathrm{~h}$

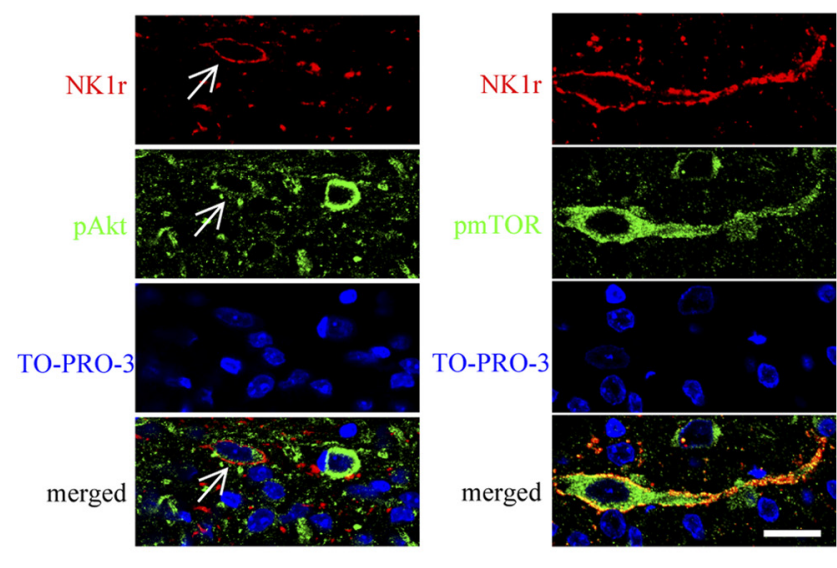

B

\section{IT SP 30min}

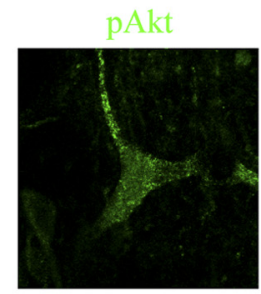

pmTOR

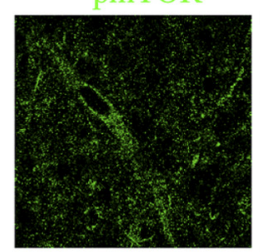

NK1r

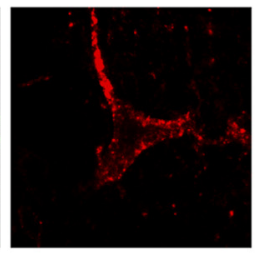

NK1r

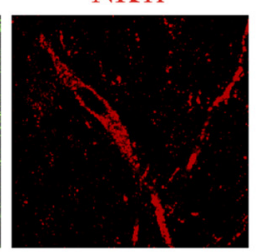

NK1r
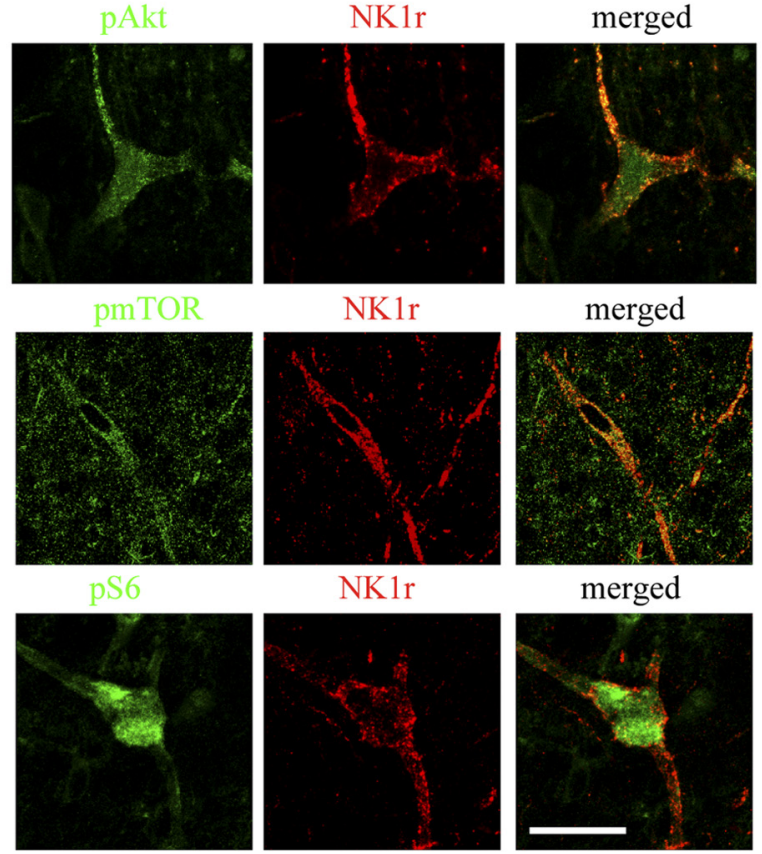

merged

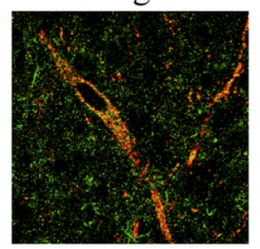

merged

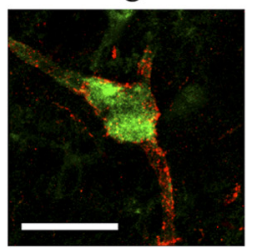

Figure 11. pAkt, pmTOR, and pS6 are colocalized with NK1R in laminae I, III, and IV. $\boldsymbol{A}$, Confocal images of pAkt or pmTOR (green) and NK1R (red) at $1 \mathrm{~h}$ after carrageenan. In each case, a single NK1R-labeled neuron is seen also pAkt or pmTOR positive. Sections are counterstained with T0-PRO-3 (blue) to label cell nuclei. $\boldsymbol{B}$, Confocal images of pAkt, pmTOR, or pS6 (green) and NK1R (red) at 30 min after intrathecal SP ( $30 \mathrm{nmol})$. A large lamina III/IV neuron is shown. NK1R internalization can be seen on the dendrites and soma. Single focal plane images are shown. Scale bars, $20 \mu \mathrm{m}$.

indeed corresponds with the very prominent paw and ankle swelling and erythema noted after carrageenan injections. In contrast with the strong expression in neurons, pAkt was observed only in a small numbers of astrocytes and not at all in microglia. This neuronal profile of expression and activation is in general agreement with previous studies using intraplantar irritants (capsaicin or Formalin) as stimuli (Ma et al., 2006; Sun et al., 2006; Pezet et al., 2008; Choi et al., 2010). A diffuse pattern of pAkt immunolabeling in the dorsal horn was also observed, which we assume represents terminals derived from primary afferents, as shown previously Akt is activated in DRG neurons by depolarization, inflammation, or axotomy (Pezet et al., 2005; Shi et al., 2009). The pattern of pmTOR in spinal dorsal horn is similar to that of pAkt. Several observations, however, indicate an addi- 
A

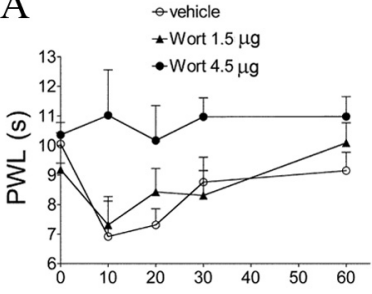

Time after IT SP (min)

C

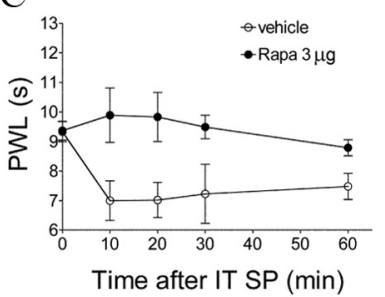

Figure 12. Effects of intrathecal wortmannin or rapamycin on spinal SP-induced pain behaviors. $A, C$, Time course over $60 \mathrm{~min}$ of PWL to thermal stimulation in the injected paw before (baseline, time 0 ) and 10, 20, 30, and $60 \mathrm{~min}$ after intrathecal (IT) SP (30 nmol). Animals received intrathecal wortmannin (Wort) $15 \mathrm{~min}$ before intrathecal SP $(\boldsymbol{A})$ or rapamycin (Rapa) $2 \mathrm{~h}$ before intrathecal $S P(\boldsymbol{C}, \boldsymbol{B}, \boldsymbol{D}$, The histograms represent the hyperalgesia index over testing period of 60 min. ${ }^{*} p<0.05$ compared with vehicle by one-way ANOVA, followed by Tukey's post hoc test $(\boldsymbol{B})$ or $t$ test $(\boldsymbol{D})$. Data are presented as mean \pm SEM; $n=7$ or 8 for each group.

tional complexity of these systems. First, there were more pmTOR-GFAP profiles than pAkt-GFAP profiles, suggesting the importance of additional mTOR signaling pathways in astrocytes, which may not be under the control of Akt. Frödin et al. (2000) showed that extracellular signal-regulated kinase (ERK) can activate mTOR. Second, these patterns represent events occurring after acute tissue injury and inflammation, and different patterns might be observed with nerve injury. Thus, pmTOR is seen in spinal astrocytes after injury (Codeluppi et al., 2009). Finally, pS6 and p4EBP were used as indicators for mTORC1 activation. The cellular distribution of pS6 primarily overlapped that of pmTOR, with the exception that pS6 was also detected in microglia. This distinction could indicate the existence of pathways other than mTORC1 that may phosphorylate and activate S6. Thus, ERK is responsible for phosphorylation of S6 in cells lacking S6K (Pende et al., 2004), possibly through p90 ribosomal protein S6 kinase (Dümmler et al., 2005). The specific antisera used for immunohistochemistry may also account for differences between reported results (Figs. 5, 6). Thus, these two antibodies may recognize different $4 \mathrm{EBP}$ isoforms.

\section{Spinal PI3K-Akt cascade and inflammatory hyperalgesia}

Intraplantar carrageenan produces a persistent thermal and tactile hyperpathia. In addition to peripheral sensitization, spinal mechanisms also play a major role in this model (Neugebauer and Schaible, 1990; Bird et al., 2006). Formalin evokes biphasic flinching, with phase I and phase II representing acute nociceptive processing and a spinally mediated facilitated state, respectively (Haley et al., 1990; Puig and Sorkin, 1996; Yaksh et al., 2001). The role of a spinal PI3K-Akt cascade in these facilitated states is indicated by the following observations. (1) Intrathecal wortmannin, a PI3K inhibitor, dose dependently attenuated carrageenan-induced thermal and tactile hyperalgesia and reversed an established thermal hyperalgesia when given as a posttreatment. Wortmannin covalently modifies PI3K and is a potent and specific PI3K inhibitor. At higher concentrations, wortman-
A

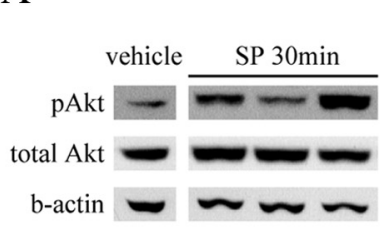

B pAkt

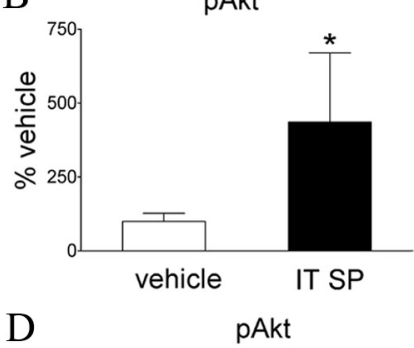

$\mathrm{C}$

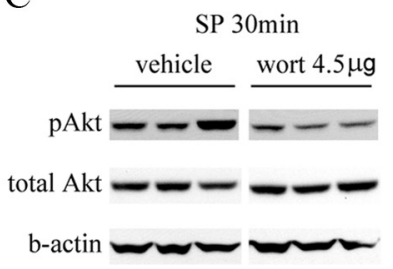

$\mathrm{E}$
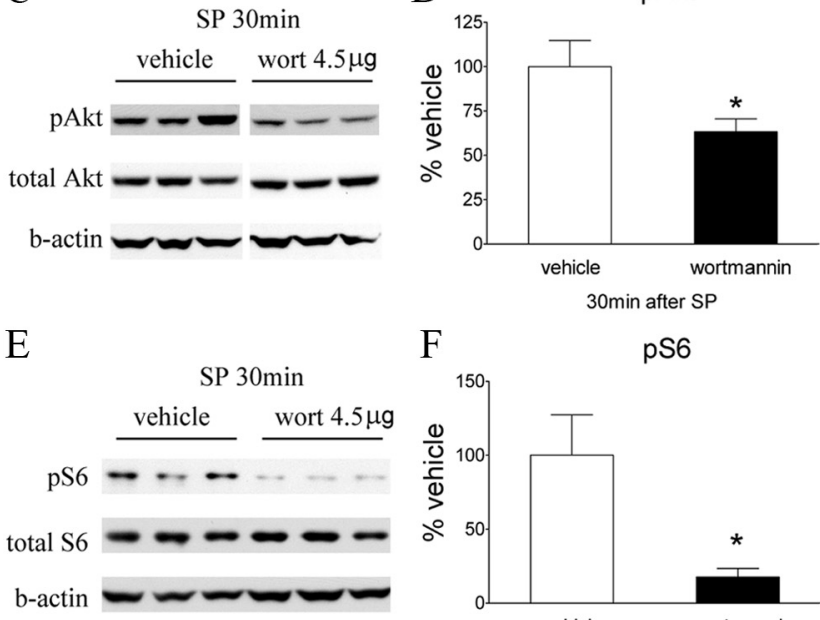

F
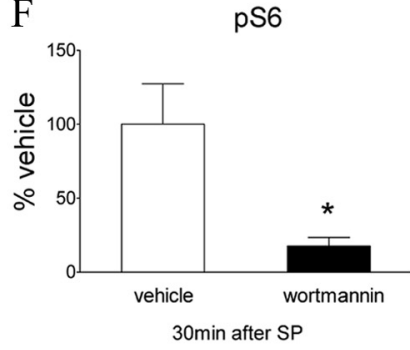

G
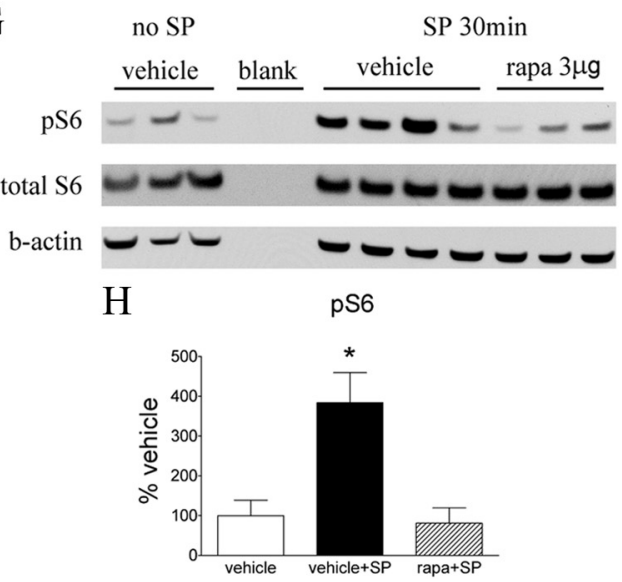

Figure 13. Effect of intrathecal wortmannin or rapamycin on intrathecal SP-induced pAkt and $p S 6$ in spinal cord assessed by Western blot. $A$, A representative blot of pAkt, total Akt, and $\beta$-actin (b-actin) in the lumbar dorsal cord at 30 min after intrathecal (IT) SP ( $30 \mathrm{nmol}$ ). Cand $\boldsymbol{E}$ show the Western blots of pAkt, total Akt, and $\beta$-actin $(\boldsymbol{C})$ and pS6, total $S 6$, and $\beta$-actin $(\boldsymbol{E})$ in the lumbar spinal dorsal cord at $30 \mathrm{~min}$ after intrathecal SP. These animals received intrathecal vehicle (3\% DMSO, $10 \mu \mathrm{l}$ ) or wortmannin (wort; $4.5 \mu \mathrm{g}$ ) 15 min before SP. G, A Western blot of $\mathrm{pS6}$, total $S 6$, and $\beta$-actin in rats that received intrathecal vehicles only, vehicle $+S P$, or rapamycin + SP. Intrathecal vehicle ( $3 \%$ DMSO, $10 \mu \mathrm{l}$ ) or rapamycin (rapa; $3 \mu \mathrm{g}$ ) was administered 15 min before SP. $\boldsymbol{B}, \boldsymbol{D}, \boldsymbol{F}, \boldsymbol{H}$, Histograms represent the mean pAkt or pS6 level with respect to the vehicle group. The data are presented as mean \pm SEM of three to six rats per group. ${ }^{*} p<0.05$ compared with vehicle by $t$ test $(\boldsymbol{B}, \boldsymbol{D}, \boldsymbol{F})$ or one-way ANOVA, followed by Tukey's post hoc test $(\boldsymbol{H})$.

nin might also inhibit myosin light chain kinase and polo-like kinase 1 (Bain et al., 2007). Our observations suggest that PI3KAkt is involved in spinal systems initiating and maintaining hyperalgesia initiated by inflammation. (2) In the Formalin model, two structurally distinct PI3K inhibitors (wortmannin, PI-103) and one Akt inhibitor (Akt-I-1/2) inhibited phase II flinching. (3) The antihyperalgesic effects were noted at doses identical to 
those, which blocked phosphorylation of the respective downstream products of this cascade (e.g., pAkt). Although intrathecal LY294002 [2-(4-morpholinyl)-8-phenyl-1(4H)-benzopyran-4one] (a PI3K inhibitor) was reported to attenuate the phase I response (Pezet et al., 2008), our data collected by an automated device did not indicate a wortmannin effect on phase I. The lack of effect on normal escape thresholds thus suggests that this cascade does not alter acute nociceptive transmission.

Involvement of spinal mTOR in PI3K-dependent nociception The present study supports involvement of mTOR in spinal PI3K-dependent nociception. Intrathecal administration of a behaviorally effective dose of wortmannin prevented the pmTOR, pS6, and p4EBP increases otherwise observed after carrageenan. The contribution of the mTORC1 to spinal sensitization is supported by the observation that intrathecal rapamycin attenuated carrageenan-induced tactile/thermal hyperalgesia and phase II Formalin flinching (Price et al., 2007; Asante et al., 2009). We note that intrathecal rapamycin displayed modest antihyperalgesic effects in these models compared with effects of intrathecal wortmannin. This difference in efficacy may reflect that PI3K is upstream to other nociceptive signaling pathways in addition to mTOR. As it is known, ERK and calcium/calmodulin-dependent kinase II are involved in PI3K-dependent spinal sensitization (Pezet et al., 2008). Thus, blockade of PI3K would generate a more robust behavioral effect reflecting on the pervasive role of this kinase in several aspects of dorsal horn sensitization evoked by peripheral tissue. Consistent with this hypothesis, spinal c-Fos expression evoked by intraplantar irritants is reversed by intrathecal PI3K inhibition (Pezet et al., 2008) but not by rapamycin (Géranton et al., 2009).

\section{The Akt-mTOR cascade in the NK1R-mediated pain circuit}

pmTOR is in superficial projection neurons (Géranton et al., 2009). Many of the projection neurons express NK1 receptors and receive dense monosynaptic inputs from SP-containing primary afferents (Todd et al., 2002; Al-Khater et al., 2008). These lamina I NK1R neurons convey nociceptive signals to medullary and brainstem regions and play a critical role in controlling dorsal horn excitability through a spino-bulbo-spinal facilitatory loop (Suzuki et al., 2002, 2005). Pharmacological elimination of NK1R-bearing neurons prevents development of central sensitization (Nichols et al., 1999; Khasabov et al., 2002; Suzuki et al., 2002; Vierck et al., 2003). In previous work, we showed that direct activation of NK1R neurons with intrathecal SP induces a centrally mediated hyperalgesia (Malmberg and Yaksh, 1992). In the present work, we show that, after intrathecal SP, strong pAkt, pmTOR, and pS6 expression were detected in dorsal horn neurons labeled with internalized NK1R (an indicator of receptor activation by SP released from primary afferents) (Mantyh et al., 1995a,b; Kondo et al., 2005). The functional relevance of this cascade is indicated by our observation that intrathecal SP-evoked hyperalgesia is blocked by wortmannin and by rapamycin. Importantly, in contrast to the modest effect on the carrageenan and Formalin-evoked hyperalgesia, rapamycin at doses that blocked $\mathrm{mTOR}$ activity produced a complete reversal of the intrathecal SP-evoked hyperalgesia, implying that enhanced transduction of nociceptive signals mediated by NK1R projection neurons represents a specific facilitatory link leading to a hyperalgesic state that is heavily dependent on the Akt-mTOR cascade. These studies thus reflect a specific neuronal linkage, the excitability of which is regulated by activation of the Akt-mTOR cascade. As represented in Figure

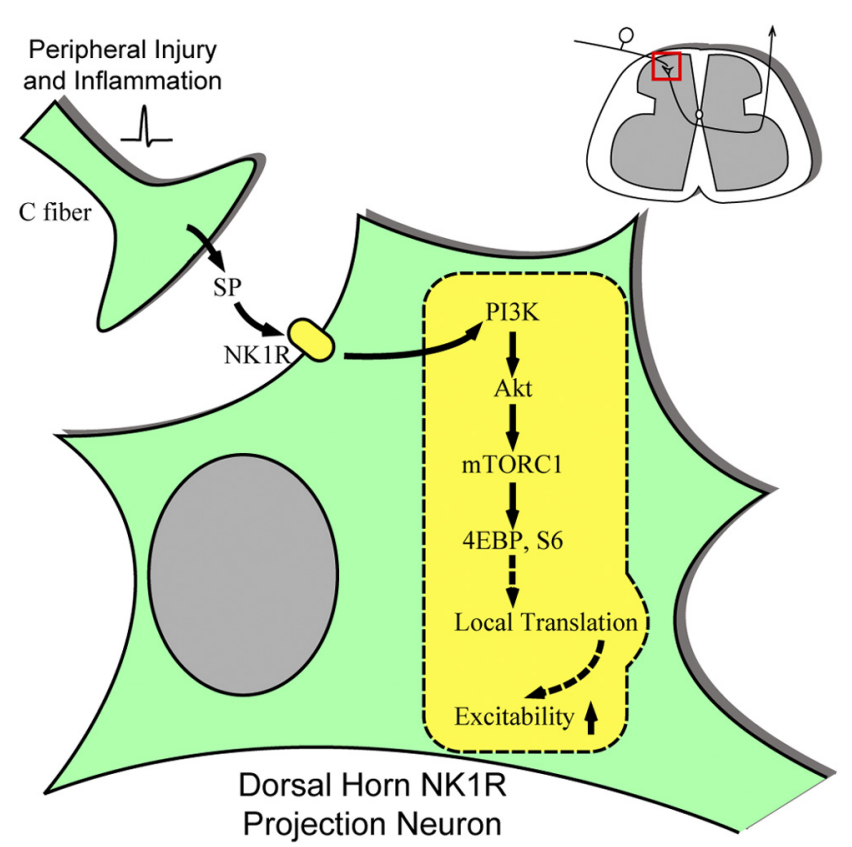

Figure 14. Schematic illustration of potential role of PI3K-Akt-mTORC1 cascade in regulating the excitability of dorsal horn neurons including NK1R projection neurons. Peripheral tissue injury and inflammation induces neurotransmitter release in the spinal cord dorsal horn, such as SP. SP activates NK1 receptors on the dorsal horn neurons, leading to the activation of the PI3K-Akt-mTORC1 cascade and phosphorylation of their substrates. mTORC1 may regulate translation of certain mRNAs, thereby enhancing the excitability of these neurons. The identities of these mRNAs are not known. Previous reports suggest certain protein kinases, ion channels, neurotropic factors, and receptors could be among the candidates (Eberwine et al., 2002; Skup, 2008).

14, such facilitation would constitute the initial component of the lamina I spino-bulbo-spinal pathway regulating dorsal horn excitability. The mechanisms underlying the neuronal effects of mTORC1 in initiating the facilitated effects have not yet been defined. We note, however, that mTOR has been convincingly shown to be involved in the initiation of rapid local protein translation processes (Richter and Sonenberg, 2005; Ma and Blenis, 2009), which have a potential role in synaptic plasticity (Huber et al., 2000; Waung et al., 2008). Several hundred species of mRNAs, the translation of which can be regulated by mTOR have been identified in hippocampal dendrites, including those that are implicated in long-term potentiation (Eberwine et al., 2002). In this regard, the fact that $\mathrm{mTORC} 1$ can regulate protein translation that takes place within minutes and contributes to synaptic plasticity is consistent with the rapidity of the time course associated with the intrathecal SP-evoked hyperalgesia. Although these comments focus on the neuronal mechanisms, the work does not exclude possible effects mediated though non-neuronal cells, nor that additional effects mediated via mechanisms other than translation may be at play in this model.

In summary, this study provides convergent biochemical, immunohistochemical, and behavioral data supporting a role for the dorsal horn PI3K-Akt-mTOR signaling pathway in the development of a facilitated pain state after peripheral inflammation. This pathway therefore links the afferent input generated by the peripheral injury to the creation of a facilitated spinal state and thereby enhances spinofugal outflow. Of equal importance, this model provides a specific locus, the NK1R dorsal horn neuron, as a link that will be useful in defining the cellular role played by this cascade leading to sensitization. 


\section{References}

Al-Khater KM, Kerr R, Todd AJ (2008) A quantitative study of spinothalamic neurons in laminae I, III, and IV in lumbar and cervical segments of the rat spinal cord. J Comp Neurol 511:1-18.

Asante CO, Wallace VC, Dickenson AH (2009) Formalin-induced behavioural hypersensitivity and neuronal hyperexcitability are mediated by rapid protein synthesis at the spinal level. Mol Pain 5:27.

Bain J, Plater L, Elliott M, Shpiro N, Hastie CJ, McLauchlan H, Klevernic I, Arthur JS, Alessi DR, Cohen P (2007) The selectivity of protein kinase inhibitors: a further update. Biochem J 408:297-315.

Bird GC, Han JS, Fu Y, Adwanikar H, Willis WD, Neugebauer V (2006) Pain-related synaptic plasticity in spinal dorsal horn neurons: role of CGRP. Mol Pain 2:31.

Chaplan SR, Bach FW, Pogrel JW, Chung JM, Yaksh TL (1994) Quantitative assessment of tactile allodynia in the rat paw. J Neurosci Methods 53:55-63.

Choi JI, Svensson CI, Koehrn FJ, Bhuskute A, Sorkin LS (2010) Peripheral inflammation induces tumor necrosis factor dependent AMPA receptor trafficking and Akt phosphorylation in spinal cord in addition to pain behavior. Pain 149:243-253.

Codeluppi S, Svensson CI, Hefferan MP, Valencia F, Silldorff MD, Oshiro M, Marsala M, Pasquale EB (2009) The Rheb-mTOR pathway is upregulated in reactive astrocytes of the injured spinal cord. J Neurosci 29:1093-1104.

Dirig DM, Salami A, Rathbun ML, Ozaki GT, Yaksh TL (1997) Characterization of variables defining hindpaw withdrawal latency evoked by radiant thermal stimuli. J Neurosci Methods 76:183-191.

Dümmler BA, Hauge C, Silber J, Yntema HG, Kruse LS, Kofoed B, Hemmings BA, Alessi DR, Frödin M (2005) Functional characterization of human RSK4, a new 90-kDa ribosomal S6 kinase, reveals constitutive activation in most cell types. J Biol Chem 280:13304-13314.

Eberwine J, Belt B, Kacharmina JE, Miyashiro K (2002) Analysis of subcellularly localized mRNAs using in situ hybridization, mRNA amplification, and expression profiling. Neurochem Res 27:1065-1077.

Frödin M, Jensen CJ, Merienne K, Gammeltoft S (2000) A phosphoserineregulated docking site in the protein kinase RSK2 that recruits and activates PDK1. EMBO J 19:2924-2934.

Géranton SM, Jiménez-Díaz L, Torsney C, Tochiki KK, Stuart SA, Leith JL, Lumb BM, Hunt SP (2009) A rapamycin-sensitive signaling pathway is essential for the full expression of persistent pain states. J Neurosci 29:15017-15027.

Haley JE, Sullivan AF, Dickenson AH (1990) Evidence for spinal $N$-methylD-aspartate receptor involvement in prolonged chemical nociception in the rat. Brain Res 518:218-226.

Hoeffer CA, Klann E (2010) mTOR signaling: at the crossroads of plasticity, memory and disease. Trends Neurosci 33:67-75.

Hou L, Klann E (2004) Activation of the phosphoinositide 3-kinase-Aktmammalian target of rapamycin signaling pathway is required for metabotropic glutamate receptor-dependent long-term depression. J Neurosci 24:6352-6361.

Huang J, Manning BD (2009) A complex interplay between Akt, TSC2 and the two mTOR complexes. Biochem Soc Trans 37:217-222.

Huber KM, Kayser MS, Bear MF (2000) Role for rapid dendritic protein synthesis in hippocampal mGluR-dependent long-term depression. Science 288:1254-1257.

Jaworski J, Sheng M (2006) The growing role of mTOR in neuronal development and plasticity. Mol Neurobiol 34:205-219.

Jiménez-Díaz L, Géranton SM, Passmore GM, Leith JL, Fisher AS, Berliocchi L, Sivasubramaniam AK, Sheasby A, Lumb BM, Hunt SP (2008) Local translation in primary afferent fibers regulates nociception. PLoS One 3:e1961.

Khasabov SG, Rogers SD, Ghilardi JR, Peters CM, Mantyh PW, Simone DA (2002) Spinal neurons that possess the substance P receptor are required for the development of central sensitization. J Neurosci 22:9086-9098.

Kondo I, Marvizon JC, Song B, Salgado F, Codeluppi S, Hua XY, Yaksh TL (2005) Inhibition by spinal mu- and delta-opioid agonists of afferentevoked substance P release. J Neurosci 25:3651-3660.

Latremoliere A, Woolf CJ (2009) Central sensitization: a generator of pain hypersensitivity by central neural plasticity. J Pain 10:895-926.

Ma W, Chabot JG, Quirion R (2006) A role for adrenomedullin as a painrelated peptide in the rat. Proc Natl Acad Sci U S A 103:16027-16032.
Ma XM, Blenis J (2009) Molecular mechanisms of mTOR-mediated translational control. Nat Rev Mol Cell Biol 10:307-318.

Malkmus SA, Yaksh TL (2004) Intrathecal catheterization and drug delivery in the rat. Methods Mol Med 99:109-121.

Malmberg AB, Yaksh TL (1992) Hyperalgesia mediated by spinal glutamate or substance P receptor blocked by spinal cyclooxygenase inhibition. Science 257:1276-1279.

Mantyh PW, Allen CJ, Ghilardi JR, Rogers SD, Mantyh CR, Liu H, Basbaum AI, Vigna SR, Maggio JE (1995a) Rapid endocytosis of a G proteincoupled receptor: substance $\mathrm{P}$ evoked internalization of its receptor in the rat striatum in vivo. Proc Natl Acad Sci U S A 92:2622-2626.

Mantyh PW, DeMaster E, Malhotra A, Ghilardi JR, Rogers SD, Mantyh CR, Liu H, Basbaum AI, Vigna SR, Maggio JE (1995b) Receptor endocytosis and dendrite reshaping in spinal neurons after somatosensory stimulation. Science 268:1629-1632.

Neugebauer V, Schaible HG (1990) Evidence for a central component in the sensitization of spinal neurons with joint input during development of acute arthritis in cat's knee. J Neurophysiol 64:299-311.

Nichols ML, Allen BJ, Rogers SD, Ghilardi JR, Honore P, Luger NM, Finke MP, Li J, Lappi DA, Simone DA, Mantyh PW (1999) Transmission of chronic nociception by spinal neurons expressing the substance $\mathrm{P}$ receptor. Science 286:1558-1561.

Norsted Gregory E, Codeluppi S, Gregory JA, Steinauer J, Svensson CI (2010) Mammalian target of rapamycin in spinal cord neurons mediates hypersensitivity induced by peripheral inflammation. Neuroscience 169:1392-1402.

Pearce LR, Komander D, Alessi DR (2010) The nuts and bolts of AGC protein kinases. Nat Rev Mol Cell Biol 11:9-22.

Pende M, Um SH, Mieulet V, Sticker M, Goss VL, Mestan J, Mueller M, Fumagalli S, Kozma SC, Thomas G (2004) S6K1-/-/S6K2-/- mice exhibit perinatal lethality and rapamycin-sensitive 5 '-terminal oligopyrimidine mRNA translation and reveal a mitogen-activated protein kinasedependent S6 kinase pathway. Mol Cell Biol 24:3112-3124.

Pezet S, Spyropoulos A, Williams RJ, McMahon SB (2005) Activitydependent phosphorylation of Akt/PKB in adult DRG neurons. Eur J Neurosci 21:1785-1797.

Pezet S, Marchand F, D’Mello R, Grist J, Clark AK, Malcangio M, Dickenson AH, Williams RJ, McMahon SB (2008) Phosphatidylinositol 3-kinase is a key mediator of central sensitization in painful inflammatory conditions. J Neurosci 28:4261-4270.

Piercey MF, Schroeder LA, Folkers K, Xu JC, Horig J (1981) Sensory and motor functions of spinal cord substance P. Science 214:1361-1363.

Price TJ, Rashid MH, Millecamps M, Sanoja R, Entrena JM, Cervero F (2007) Decreased nociceptive sensitization in mice lacking the fragile $\mathrm{X}$ mental retardation protein: role of mGluR1/5 and mTOR. J Neurosci 27:13958-13967.

Puig S, Sorkin LS (1996) Formalin-evoked activity in identified primary afferent fibers: systemic lidocaine suppresses phase-2 activity. Pain 64:345-355.

Richter JD, Sonenberg N (2005) Regulation of cap-dependent translation by eIF4E inhibitory proteins. Nature 433:477-480.

Shi TJ, Huang P, Mulder J, Ceccatelli S, Hokfelt T (2009) Expression of p-Akt in sensory neurons and spinal cord after peripheral nerve injury. Neurosignals 17:203-212.

Skup M (2008) Dendrites as separate compartment: local protein synthesis. Acta Neurobiol Exp (Wars) 68:305-321.

Sun RQ, Tu YJ, Yan JY, Willis WD (2006) Activation of protein kinase B/Akt signaling pathway contributes to mechanical hypersensitivity induced by capsaicin. Pain 120:86-96.

Sun R, Yan J, Willis WD (2007) Activation of protein kinase B/Akt in the periphery contributes to pain behavior induced by capsaicin in rats. Neuroscience 144:286-294.

Suzuki R, Rahman W, Rygh LJ, Webber M, Hunt SP, Dickenson AH (2005) Spinal-supraspinal serotonergic circuits regulating neuropathic pain and its treatment with gabapentin. Pain 117:292-303.

Suzuki R, Morcuende S, Webber M, Hunt SP, Dickenson AH (2002) Superficial NK1-expressing neurons control spinal excitability through activation of descending pathways. Nat Neurosci 5:1319-1326.

Swett JE, Woolf CJ (1985) The somatotopic organization of primary afferent terminals in the superficial laminae of the dorsal horn of the rat spinal cord. J Comp Neurol 231:66-77.

Todd AJ, Puskar Z, Spike RC, Hughes C, Watt C, Forrest L (2002) Projec- 
tion neurons in lamina I of rat spinal cord with the neurokinin 1 receptor are selectively innervated by substance p-containing afferents and respond to noxious stimulation. J Neurosci 22:4103-4113.

Vierck CJ Jr, Kline RH, Wiley RG (2003) Intrathecal substance p-saporin attenuates operant escape from nociceptive thermal stimuli. Neuroscience 119:223-232.

Waung MW, Pfeiffer BE, Nosyreva ED, Ronesi JA, Huber KM (2008) (Rapid translation of Arc/Arg3.1 selectively mediates mGluR-dependent LTD through persistent increases in AMPAR endocytosis rate. Neuron 59:84-97.

Xu JT, Tu HY, Xin WJ, Liu XG, Zhang GH, Zhai CH (2007 Activation of phosphatidylinositol 3-kinase and protein kinase B/Akt in dorsal root ganglia and spinal cord contributes to the neuropathic pain induced by spinal nerve ligation in rats. Exp Neurol 206:269-279.

Xu JT, Zhao X, Yaster M, Tao YX (2010) Expression and distribution of
mTOR, p70S6K, 4E-BP1, and their phosphorylated counterparts in rat dorsal root ganglion and spinal cord dorsal horn. Brain Res 1336:46-57.

Yaksh TL, Rudy TA (1976) Chronic catheterization of the spinal subarachnoid space. Physiol Behav 17:1031-1036.

Yaksh TL, Ozaki G, McCumber D, Rathbun M, Svensson C, Malkmus S, Yaksh MC (2001) An automated flinch detecting system for use in the formalin nociceptive bioassay. J Appl Physiol 90:2386-2402.

Yang Q, Guan KL (2007) Expanding mTOR signaling. Cell Res 17:666-681.

Yashpal K, Henry JL (1983) Endorphins mediate overshoot of substance P-induced facilitation of a spinal nociceptive reflex. Can J Physiol Pharmacol 61:303-307.

Zhuang ZY, Xu H, Clapham DE, Ji RR (2004) Phosphatidylinositol 3-kinase activates ERK in primary sensory neurons and mediates inflammatory heat hyperalgesia through TRPV1 sensitization. J Neurosci 24:83008309. 